\title{
A new class of hyperbolic variational-hemivariational inequalities driven by non-linear evolution equations $\dagger$
}

\author{
STANIS ŁAW MIGÓRSKI ${ }^{1,2,3}$, WEIMIN HAN ${ }^{4}$ and SHENGDA ZENG ${ }^{2,3}$ \\ ${ }^{1}$ College of Applied Mathematics, Chengdu University of Information Technology, \\ Chengdu 610225, Sichuan Province, P.R. China \\ email: stanislaw.migorski@uj.edu.pl \\ ${ }^{2}$ Guangxi Colleges and Universities Key Laboratory of Complex System Optimization and \\ Big Data Processing, Yulin Normal University, Yulin 537000, P.R. China \\ ${ }^{3}$ Jagiellonian University in Krakow, ul. Lojasiewicza 6, 30348 Krakow, Poland \\ emails: shengdazeng@gmail.com; shdzeng@hotmail.com; zengshengda@163.com \\ ${ }^{4}$ Department of Mathematics, University of Iowa, Iowa City, IA 52242-1410, USA \\ email:weimin-han@uiowa.edu
}

(Received 8 July 2019; revised 8 December 2019; accepted 12 February 2020; first published online 16 March 2020)

\begin{abstract}
The aim of the paper is to introduce and investigate a dynamical system which consists of a variational-hemivariational inequality of hyperbolic type combined with a non-linear evolution equation. Such a dynamical system arises in studies of complicated contact problems in mechanics. Existence, uniqueness and regularity of a global solution to the system are established. The approach is based on a new semi-discrete approximation with an application of a surjectivity result for a pseudomonotone perturbation of a maximal monotone operator. A new dynamic viscoelastic frictional contact model with adhesion is studied as an application, in which the contact boundary condition is described by a generalised normal damped response condition with unilateral constraint and a multivalued frictional contact law.
\end{abstract}

Key words: Hyperbolic variational-hemivariational inequality, Clarke subgradient, existence and uniqueness, dynamic contact problem, normal damped response, adhesion

2010 Mathematics Subject Classification: 49J40, 47J20, 47J22 (Primary); 74M10, 74M15, 74G25, 35L51 (Secondary)

\section{Introduction}

In real life, a wide variety of physical phenomena, economic processes and engineering applications are naturally modelled or formulated as inequality problems which replace more commonly

$\dagger$ This project has received funding from the European Union's Horizon 2020 Research and Innovation Programme under the Marie Skłodowska-Curie grant agreement no. 823731 CONMECH. It is supported by the National Science Center of Poland under Preludium project no. 2017/25/N/ST1/00611. The first author is also supported by the Natural Science Foundation of Guangxi grant no. 2018GXNSFAA281353 and the Ministry of Science and Higher Education of Republic of Poland under grants no. 4004/GGPJII/H2020/2018/0 and 440328/PnH2/2019. The work of the second author was partially supported by NSF under grant DMS- 1521684 . 
studied equations. On the one hand, physical laws and constitutive relations for complicated applications are often described by inequalities, and on the other hand, investigation of inequality problems is more difficult than equations. Roughly speaking, inequality problems can be classified as variational inequalities and hemivariational inequalities. Variational inequalities are related to convex potentials and their study is traced back to 1933 when Signorini posed a static linear elastic contact problem, which was subsequently studied by Fichera in 1964 on solution existence in the space of functions with finite energy by exploiting an optimisation method. Over half a century, variational inequalities have attracted much attention due to their wide range of applications in Mechanics, Engineering, Economics, etc., see, for example, [11, 12, 22, 27, 28, 30, $31,38]$. The theory of hemivariational inequalities is based on the notion of the Clarke's generalised subdifferential defined for locally Lipschitz functions, and it was started with works of Panagiotopoulos in the early 1980s, see [40], who attempted to analyse engineering problems involving non-smooth, non-monotone and possibly multivalued constitutive/interface laws for deformable bodies. Since then, there has been much development on the mathematical theory and applications of hemivariational inequalities, see, for example, [6, 24, 35, 37, 39, 41, 46, 49].

Although the notion of variational-hemivariational inequality is not new, see, for example, [42], there is extensive research on variational-hemivariational inequalities in the recent years to explore complicated systems involving both convex and non-convex energy (or potential) functions. Among recent results, we mention some representative references: [19] on existence and uniqueness result for a class of variational-hemivariational inequalities of elliptic type and optimal-order error estimate for the linear finite element approximations; [4, 5] on the existence of solution to parabolic variational-hemivariational inequalities; [17] treated an adhesive unilateral contact problem between a viscoelastic body and a deformable foundation which can be formulated as a dynamical system consisting of a variational-hemivariational inequality of parabolic type and an ordinary differential equation; [32] built a Landesman-Lazer theory in the non-smooth framework of variational-hemivariational inequalities of elliptic type; and [23] on the numerical solution of elliptic variational-hemivariational inequalities.

The aim of this paper is to study a new class of dynamical system consisting of a hyperbolic variational-hemivariational inequality and a non-linear evolution equation. This system is formulated as follows. Given Banach spaces $V, X, Y$, operators $A: V \rightarrow V^{*}, B: V \rightarrow V^{*}, M: V \rightarrow X$, a locally Lipschitz function $J: Y \times X \rightarrow \mathbb{R}$, a function $F:(0, T) \times X \times Y \rightarrow Y$, a proper, convex and lower semicontinuous function $\varphi: V \rightarrow \overline{\mathbb{R}}:=\mathbb{R} \cup\{+\infty\}, f \in \mathcal{V}^{*}, u_{0}, v_{0} \in V$ and $\beta_{0} \in Y$, we are looking for functions $u:(0, T) \rightarrow V, \beta:(0, T) \rightarrow Y$ and $\xi:(0, T) \rightarrow X^{*}$ such that

$$
\left\{\begin{array}{l}
\left\langle u^{\prime \prime}(t), v-u^{\prime}(t)\right\rangle+\left\langle A u^{\prime}(t)+B u(t), v-u^{\prime}(t)\right\rangle+\varphi(v)-\varphi\left(u^{\prime}(t)\right) \\
\quad+\left\langle\xi(t), M\left(v-u^{\prime}(t)\right)\right\rangle_{X^{*} \times X} \geq\left\langle f(t), v-u^{\prime}(t)\right\rangle \quad \text { for all } v \in V, \text { a.e. } t \in(0, T), \\
\xi(t) \in \partial J\left(\beta(t), M u^{\prime}(t)\right) \quad \text { for a.e. } t \in(0, T), \\
\beta^{\prime}(t)=F(t, M u(t), \beta(t)) \quad \text { for a.e. } t \in(0, T), \\
u(0)=u_{0}, \quad u^{\prime}(0)=v_{0}, \quad \beta(0)=\beta_{0} .
\end{array}\right.
$$

Such a dynamical system arises in studies of complicated contact problems in mechanics in which the non-linear evolution equation models the adhesion effect. Existence and uniqueness of solution will be proved for the system, based on a new semi-discrete approximation of the system together with the application of a surjectivity result for a pseudomonotone perturbation of a maximal monotone operator. The system (1.1) has been studied earlier in [36] with $\varphi \equiv 0$ and 
$J$ depending on $u$. Since the function $\varphi$ takes infinite values, the results for (1.1) are applicable to unilateral contact problems. This application was not possible for the problem treated in [36]. Moreover, problem (1.1) was considered in [3] without the non-linear evolution equation and under more restrictive hypotheses on the data.

The outline of the paper is as follows: basic notation and preliminary materials needed in the rest of the paper are recalled in Section 2. In Section 3, we provide an existence and uniqueness analysis of the dynamical system. We apply a new temporally semi-discretisation of mixed type to approximate the dynamical system. Solution existence of the semi-discrete scheme is obtained by applying a general surjectivity result for pseudomonotone perturbations of maximal monotone operators. Then, a priori bounds are established on solutions of the semi-discrete approximations, and piecewise affine and piecewise constant interpolation functions are constructed. Through a limiting procedure, we show that the semi-discrete solutions converge to a solution of the dynamical system. Solution uniqueness is also examined. In Section 4, a new dynamic viscoelastic contact model with combined effects of friction and adhesion is studied as an illustrative application. We provide a weak formulation of the contact problem in the form of hyperbolic variational-hemivariational inequality coupled with a non-linear evolution equation and apply the theoretical results from Section 3 to conclude the existence of a unique weak solution of the contact problem.

\section{Notation and preliminaries}

In this section, we collect basic notation and some results needed later. For more details, we refer to $[8-10,48]$.

Throughout the paper, we denote by $\langle\cdot, \cdot\rangle_{Y^{*} \times Y}$ the duality pairing between a Banach space $Y$ and its dual $Y^{*}$. The norm in a normed space $Y$ is denoted by $\|\cdot\|_{Y}$. We often drop the subscripts, if no confusion arises. We denote by $\mathcal{L}\left(Y_{1}, Y_{2}\right)$ the space of linear and continuous operators from a normed space $Y_{1}$ to a normed space $Y_{2}$ endowed with the operator norm $\|\cdot\|_{\mathcal{L}\left(Y_{1}, Y_{2}\right)}$. For an operator $A \in \mathcal{L}\left(Y_{1}, Y_{2}\right)$, we denote by $A^{*} \in \mathcal{L}\left(Y_{2}^{*}, Y_{1}^{*}\right)$ its adjoint.

Given a reflexive Banach space $X$, and a multivalued operator $A: X \rightarrow 2^{X^{*}}$, we say that $A$ is pseudomonotone, if

(i) for each $u \in X$, the set $A u$ is non-empty, bounded, closed and convex in $X^{*}$;

(ii) $A$ is upper semicontinuous from each finite-dimensional subspace of $X$ to $X^{*}$ endowed with weak $*$ topology;

(iii) if $\left\{v_{n}\right\} \subset X$ and $\left\{v_{n}^{*}\right\} \subset X^{*}$ are such that $v_{n} \rightarrow v$ weakly in $X$ and $v_{n}^{*} \in A\left(v_{n}\right)$ with $\lim \sup _{n \rightarrow \infty}\left\langle v_{n}^{*}, v_{n}-v\right\rangle \leq 0$, then for each $u \in X$ there exists $v^{*}(u) \in A(v)$ such that

$$
\left\langle v^{*}(u), v-u\right\rangle \leq \liminf _{n \rightarrow \infty}\left\langle v_{n}^{*}, v_{n}-u\right\rangle
$$

A single-valued mapping $A: X \rightarrow X^{*}$ is called pseudomonotone, if it is bounded and if for every sequence $\left\{v_{n}\right\} \subseteq X$ converging weakly to $v \in X$ and $\lim \sup \left\langle A v_{n}, v_{n}-v\right\rangle \leq 0$, one has $\langle A v, v-u\rangle \leq \liminf _{n \rightarrow \infty}\left\langle A v_{n}, v_{n}-u\right\rangle$ for all $u \in X$. It is known, see [35, Proposition 3.66], that $A$ is pseudomonotone if and only if the following holds: $v_{n} \rightarrow v$ weakly in $X$ and $\lim \sup \left\langle A v_{n}, v_{n}-v\right\rangle \leq 0$ entails $\lim _{n \rightarrow \infty}\left\langle A v_{n}, v_{n}-v\right\rangle=0$ and $A v_{n} \rightarrow A v$ weakly in $X^{*}$. It is clear that if $A \in \mathcal{L}\left(X, X^{*}\right)$ is non-negative, then it is pseudomonotone. 
Let $\varphi: X \rightarrow \overline{\mathbb{R}}:=\mathbb{R} \cup\{+\infty\}$. We say that $\varphi$ is proper, if its effective domain dom $\varphi=\{v \in$ $X \mid \varphi(v)<+\infty\} \neq \emptyset$. The function $\varphi$ is (sequentially) lower semicontinuous (1.s.c., for short), if $v_{n} \rightarrow v$ in $X$, as $n \rightarrow+\infty$ implies $\varphi(v) \leq \lim \inf \varphi\left(v_{n}\right)$. For a proper, convex and 1.s.c. function $\varphi: X \rightarrow \overline{\mathbb{R}}$, its convex subdifferential is a multivalued mapping $\partial_{c} \varphi: X \rightarrow 2^{X^{*}}$ defined by

$$
\partial_{c} \varphi(u)=\left\{u^{*} \in X^{*} \mid\left\langle u^{*}, v-u\right\rangle \leq \varphi(v)-\varphi(u) \text { for all } v \in X\right\} .
$$

The elements of the set $\partial_{c} \varphi(u)$ are called (convex) subgradients of $\varphi$ at $u \in X$.

A function $J: X \rightarrow \mathbb{R}$ is called locally Lipschitz at $u \in X$, if there exists a neighbourhood $O_{u}$ of $u$ and a constant $L_{u}>0$ such that $|J(w)-J(v)| \leq L_{u}\|w-v\|_{X}$ for all $w, v \in O_{u}$. Given a locally Lipschitz function $J: X \rightarrow \mathbb{R}$, its generalised (Clarke) directional derivative at the point $u \in X$ in the direction $v \in X$ is defined by

$$
J^{0}(u ; v)=\limsup _{\lambda \rightarrow 0^{+}, w \rightarrow u} \frac{J(w+\lambda v)-J(w)}{\lambda} .
$$

The generalised gradient of $J: X \rightarrow \mathbb{R}$ at point $u \in X$ is given by $\partial J(u)=\left\{\xi \in X^{*} \mid J^{0}(u ; v) \geq\right.$ $\langle\xi, v\rangle$ for all $v \in X\}$.

A function $h: X \rightarrow \mathbb{R}$ is (sequentially) upper semicontinuous (u.s.c., for short), if $v_{n} \rightarrow v$ in $X$, as $n \rightarrow+\infty$ implies lim $\sup h\left(v_{n}\right) \leq h(v)$.

The next proposition, see [20, Proposition 5.6, p. 114], provides an example of a multivalued pseudomonotone operator corresponding to superposition of the generalised subgradient with a compact operator.

Proposition 1 Let $V$ and $X$ be reflexive Banach spaces. Let $M: V \rightarrow X$ be a linear, continuous and compact operator with its adjoint $M^{*}: X^{*} \rightarrow V^{*}$, and $J: X \rightarrow \mathbb{R}$ be a locally Lipschitz function such that $\|\partial J(v)\|_{X^{*}} \leq c\left(1+\|v\|_{X}\right)$ for all $v \in V$ with $c>0$. Then, the multivalued operator $F: V \rightarrow 2^{V^{*}}$ defined by $F(v)=M^{*} \partial J(M v)$ for $v \in V$ is pseudomonotone.

Let $T \in(0, \infty)$ and $q \in[1, \infty)$ be given. Denote by $\pi$ a finite partition of the interval $[0, T]$ by a family of disjoint subintervals $\Delta_{i}:=\left[l_{i}, r_{i}\right]$ such that $[0, T]=\cup_{i=1}^{n} \Delta_{i}$. Let $\mathscr{P}$ denote the family of all such partitions. Furthermore, we introduce the space $B V^{q}(0, T ; X):=\{x:(0, T) \rightarrow$ $\left.X \mid\|x\|_{B V^{q}(0, T ; X)}<\infty\right\}$, where $\|x\|_{B V^{q}(0, T ; X)}$ stands for the seminorm of $x \in B V^{q}(0, T ; X)$ given by

$$
\|x\|_{B V^{q}(0, T ; X)}^{q}:=\sup _{\pi \in \mathscr{P}} \sum_{\Delta_{i} \in \pi}\left\|x\left(r_{i}\right)-x\left(l_{i}\right)\right\|_{X}^{q} .
$$

Let $X$ and $Z$ be Banach spaces with the continuous embedding $X \subset Z$. For $1 \leq p, q<\infty$ fixed, the space $M^{p, q}(0, T ; X, Z)=L^{p}(0, T ; X) \cap B V^{q}(0, T ; Z)$ becomes a Banach space under the norm $\|\cdot\|_{M^{p, q}(0, T ; X, Z)}:=\|\cdot\|_{L^{p}(0, T ; X)}+\|\cdot\|_{B V^{q}(0, T ; Z)}$. The following compactness result will be needed, see [26, Proposition 2.8].

Proposition 2 Let $1 \leq p, q<\infty$ and $X_{1} \subset X_{2} \subset X_{3}$ be Banach spaces such that $X_{1}$ is reflexive, the embedding $X_{1} \subset X_{2}$ is compact and the embedding $X_{2} \subset X_{3}$ is continuous. Then, any bounded subset of $M^{p, q}\left(0, T ; X_{1}, X_{3}\right)$ is relatively compact in $L^{p}\left(0, T ; X_{2}\right)$.

The following result provides a surjectivity criterion for an operator which is the sum of a pseudomonotone and a maximal monotone operator, see [29, Theorem 2.2]. 
Theorem 3 Let $\mathcal{F}: D(\mathcal{F}) \subset X \rightarrow 2^{X^{*}}$ be a maximal monotone operator, $\mathcal{G}: D(\mathcal{G})=X \rightarrow 2^{X^{*}}$ a bounded multivalued pseudomonotone operator and $L \in X^{*}$. Assume that there exist $u_{0} \in X$ and $R \geq\left\|u_{0}\right\|_{X}$ such that $D(\mathcal{F}) \cap B_{R}\left(0_{X}\right) \neq \emptyset$ and

$$
\left\langle\xi+\eta-L, u-u_{0}\right\rangle>0,
$$

for all $u \in D(\mathcal{F})$ with $\|u\|_{X}=R$ and all $\xi \in \mathcal{F}(u), \eta \in \mathcal{G}(u)$, where $B_{R}\left(0_{X}\right)$ stands for the open ball centred at $0_{X}$ with radius $R$. Then, there exists an element $u \in D(\mathcal{F})$ such that $\mathcal{F}(u)+\mathcal{G}(u) \ni L$.

In the proofs of next section, we will apply the following modified Cauchy-Schwarz inequality on several occasions: for any reals $a$ and $b$, and any $\varepsilon>0$,

$$
a b \leq \varepsilon a^{2}+C b^{2} \quad \text { with } C=1 /(4 \varepsilon) .
$$

\section{Variational-hemivariational inequalities of hyperbolic type}

In this section, we study a new class of dynamical systems which consists of a generalised hyperbolic variational-hemivariational inequality coupled with a non-linear evolution equation. Results on existence, uniqueness and regularity of solution to the dynamical system are provided.

The functional framework used in this section is the following (we refer to $[9,10,48]$ for details). Let $V \subset H \subset V^{*}$ be an evolution triple of spaces, that is, $(V,\|\cdot\|)$ is a reflexive and separable Banach space with its dual $\left(V^{*},\|\cdot\|_{V^{*}}\right),\left(H,\|\cdot\|_{H}\right)$ is a separable Hilbert space and the embedding of $V$ into $H$ is dense and continuous. Moreover, we assume that the embedding operator $i: V \rightarrow H$ is compact. The duality pairing between $V^{*}$ and $V$ and the scalar product of $H$ are denoted by $\langle\cdot, \cdot\rangle$ and $(\cdot, \cdot)_{H}$, respectively. Given $T \in(0, \infty)$, we consider the standard Bochner-Lebesgue function spaces

$$
\mathcal{V}=L^{2}(0, T ; V), \mathcal{H}=L^{2}(0, T ; H) \text { and } \mathcal{W}=\left\{v \in \mathcal{V} \mid v^{\prime} \in \mathcal{V}^{*}\right\}
$$

where the time derivative $v^{\prime}=\partial v / \partial t$ is understood in the sense of vector-valued distributions. Note that spaces $\mathcal{V}$ and $\mathcal{V}^{*}=L^{2}\left(0, T ; V^{*}\right)$ are reflexive Banach spaces, $\mathcal{W}$ endowed with graph norm $\|v\|_{\mathcal{W}}=\|v\|_{\mathcal{V}}+\left\|v^{\prime}\right\|_{\mathcal{V}^{*}}$ is a separable and reflexive Banach space, and the embeddings $\mathcal{W} \subset \mathcal{V} \subset \mathcal{H} \subset \mathcal{V}^{*}$ are continuous. Also, the embedding $\mathcal{W} \subset C(0, T ; H)$ is continuous, where $C(0, T ; H)$ stands for the space of continuous functions on $[0, T]$ with values in $H$.

Furthermore, assume that $\left(X,\|\cdot\|_{X}\right)$ and $\left(Y,\|\cdot\|_{Y}\right)$ are two Banach spaces with duals $\left(X^{*},\|\cdot\|_{X^{*}}\right)$ and $\left(Y^{*},\|\cdot\|_{Y^{*}}\right)$, respectively, the duality pairing for $X$ being denoted by $\langle\cdot, \cdot\rangle_{X^{*} \times X}$. Additionally, we introduce function spaces $\mathcal{Y}=L^{2}(0, T ; Y), \mathcal{Y}^{*}=L^{2}\left(0, T ; Y^{*}\right), \mathcal{X}=L^{2}(0, T ; X)$ and $\mathcal{X}^{*}=L^{2}\left(0, T ; X^{*}\right)$. We use the notations $\langle\cdot, \cdot\rangle_{\mathcal{V}^{*} \times \mathcal{V}}$ and $\langle\cdot, \cdot\rangle_{\mathcal{X}^{*} \times \mathcal{X}}$ for the dualities between $\mathcal{V}$ and $\mathcal{V}^{*}$, and $\mathcal{X}$ and $\mathcal{X}^{*}$, respectively.

In the rest of the paper, we denote by $C$ a generic constant whose value may change from line to line.

Given a non-linear operator $A: V \rightarrow V^{*}$, a linear operator $B: V \rightarrow V^{*}$, a locally Lipschitz function $J: Y \times X \rightarrow \mathbb{R}$, a non-linear function $F:(0, T) \times X \times Y \rightarrow Y$, a proper, convex and lower semicontinuous function $\varphi: V \rightarrow \overline{\mathbb{R}}:=\mathbb{R} \cup\{+\infty\}$, a linear operator $M: V \rightarrow X$, elements $f \in \mathcal{V}^{*}, u_{0}, v_{0} \in V$ and $\beta_{0} \in Y$, the goal of this paper is to study the following system of a generalised hyperbolic variational-hemivaritional inequality coupled with a non-linear evolution equation. 
Problem 4 Find $(u, \beta) \in \mathcal{V} \times W^{1,2}(0, T ; Y)$ with $u^{\prime} \in \mathcal{W}$ such that there exists $\xi \in \mathcal{X}^{*}$ and

$$
\left\{\begin{array}{l}
\left\langle u^{\prime \prime}(t), v-u^{\prime}(t)\right\rangle+\left\langle A u^{\prime}(t)+B u(t), v-u^{\prime}(t)\right\rangle+\varphi(v)-\varphi\left(u^{\prime}(t)\right) \\
\quad+\left\langle\xi(t), M\left(v-u^{\prime}(t)\right)\right\rangle_{X^{*} \times X} \geq\left\langle f(t), v-u^{\prime}(t)\right\rangle \quad \text { for all } v \in V, \text { a.e. } t \in(0, T), \\
\xi(t) \in \partial J\left(\beta(t), M u^{\prime}(t)\right) \quad \text { for a.e. } t \in(0, T), \\
\beta^{\prime}(t)=F(t, M u(t), \beta(t)) \quad \text { for a.e. } t \in(0, T), \\
u(0)=u_{0}, \quad u^{\prime}(0)=v_{0}, \quad \beta(0)=\beta_{0} .
\end{array}\right.
$$

We impose the following assumptions on the data of Problem 4.

$H(A)$ : The operator $A: V \rightarrow V^{*}$ is such that

(i) $A$ is pseudomonotone.

(ii) There exist constants $a_{A} \geq 0$ and $b_{A}>0$ satisfying

$$
\|A v\|_{V^{*}} \leq a_{A}+b_{A}\|v\| \text { for all } v \in V .
$$

(iii) There exist $m_{A}>0$ and $c_{A} \geq 0$ such that the inequality is true

$$
\langle A u-A v, u-v\rangle \geq m_{A}\|u-v\|^{2}-c_{A}\|u-v\|_{H}^{2} \text { for all } u, v \in V .
$$

$H(B)$ : The operator $B \in \mathcal{L}\left(V, V^{*}\right)$ is symmetric and coercive, that is, there exists a constant $\varrho_{B}>0$ such that

$$
\langle B u, v\rangle=\langle B v, u\rangle \text { and }\langle B u, u\rangle \geq \varrho_{B}\|u\|^{2} \text { for all } u, v \in V .
$$

$H(f): f \in H^{1}\left(0, T ; V^{*}\right)$.

$H(J)$ : The function $J: Y \times X \rightarrow \mathbb{R}$ is such that

(i) $x \mapsto J(y, x)$ is locally Lipschitz for all $y \in Y$.

(ii) The growth condition holds

$$
\|\partial J(y, x)\|_{X^{*}} \leq c_{J}\left(1+\|x\|_{X}\right) \quad \text { for all } x \in X, y \in Y \text { with some } c_{J}>0 .
$$

(iii) There exists $m_{J} \geq 0$ such that for all $\xi_{i} \in \partial J\left(y_{i}, x_{i}\right)$ and $\left(y_{i}, x_{i}\right) \in Y \times X, i=1,2$,

$$
\left\langle\xi_{1}-\xi_{2}, x_{1}-x_{2}\right\rangle_{X^{*} \times X} \geq-m_{J}\left(\left\|x_{1}-x_{2}\right\|_{X}+\left\|y_{1}-y_{2}\right\|_{Y}\right)\left\|x_{1}-x_{2}\right\|_{X} .
$$

(iv) $(y, x) \mapsto J^{0}(y, x ; z)$ is u.s.c. from $Y \times X$ into $\mathbb{R}$ for all $z \in X$.

$H(\varphi)$ : The function $\varphi: V \rightarrow \overline{\mathbb{R}}:=\mathbb{R} \cup\{+\infty\}$ is proper, convex and 1.s.c.

$H(M)$ : The operator $M: V \rightarrow X$ is linear, continuous, and its Nemytskii operator $\mathcal{M}: M^{2,2}\left(0, T ; V, V^{*}\right) \subset \mathcal{V} \rightarrow \mathcal{X}$ is compact, where $\mathcal{M}$ is defined by

$$
(\mathcal{M} v)(t)=M(v(t)) \text { for a.e. } t \in(0, T) \text { and } v \in \mathcal{V} .
$$

$H(F): F \in L^{\infty}([0, T] \times X \times Y ; Y)$ with $M_{F}:=$ ess sup $\|F(\cdot, \cdot, \cdot)\|_{Y}<\infty$ and

(i) $t \mapsto F(t, x, y)$ is measurable on $(0, T)$ for all $x \in X, y \in Y$.

(ii) $(x, y) \mapsto F(t, x, y)$ is Lipschitz continuous for a.e. $t \in(0, T)$ with a Lipschitz constant $L_{F}>0$, 
that is, for any $\left(x_{1}, y_{1}\right),\left(x_{2}, y_{2}\right) \in X \times Y$, it holds

$$
\left\|F\left(t, x_{1}, y_{1}\right)-F\left(t, x_{2}, y_{2}\right)\right\|_{Y} \leq L_{F}\left(\left\|x_{1}-x_{2}\right\|_{X}+\left\|y_{1}-y_{2}\right\|_{Y}\right) \text { for a.e. } t \in(0, T) .
$$

$H(0)$ : The following compatibility and smallness conditions are satisfied:

(i) $u_{0} \in V, v_{0} \in D\left(\partial_{c} \varphi\right), \beta_{0} \in Y$, and there exist $z_{0} \in H$ and $\xi_{0} \in \partial J\left(\beta_{0}, M v_{0}\right)$ such that for all $v \in V$, it holds

$$
\left(z_{0}, v-v_{0}\right)_{H}+\left\langle A v_{0}+B u_{0}, v-v_{0}\right\rangle+\left\langle\xi_{0}, M\left(v-v_{0}\right)\right\rangle_{X^{*} \times X}+\varphi(v)-\varphi\left(v_{0}\right) \geq\left\langle f(0), v-v_{0}\right\rangle .
$$

(ii) $m_{A}>m_{J}\|M\|_{\mathcal{L}(V, X)}^{2}$.

The following lemmata will be useful, see [36, Lemmata 7 and 8].

Lemma 5 Assume $H(J)(i)-(i i)$ and $H(J)(i v)$. Then the multivalued operator $(y, x) \mapsto \partial J(y, x)$ is upper semicontinuous from $Y \times X$ endowed with the norm topology to the subsets of $X^{*}$ endowed with the weak ${ }^{*}$ topology.

Lemma 6 Assume $H(F)$ and $\beta_{0} \in Y$. Then, for any $u \in \mathcal{X}$, there exists $\beta \in W^{1,2}(0, T ; Y) \subset$ $C(0, T ; Y)$ a unique solution to the Cauchy problem

$$
\left\{\begin{array}{l}
\beta^{\prime}(t)=F(t, u(t), \beta(t)) \quad \text { for a.e. } t \in(0, T) \\
\beta(0)=\beta_{0} .
\end{array}\right.
$$

Moreover, given $u_{i} \in \mathcal{X}$ and denoting by $\beta_{i} \in W^{1,2}(0, T ; Y)$ the unique solution to problem (3.2) corresponding to $u_{i}$, for $i=1,2$, the following inequality holds

$$
\left\|\beta_{1}(t)-\beta_{2}(t)\right\|_{Y} \leq c_{F} \int_{0}^{t}\left\|u_{1}(s)-u_{2}(s)\right\|_{X} d s,
$$

for all $t \in[0, T]$ with some $c_{F}>0$. Further, we have the estimate

$$
\left\|\beta_{1}-\beta_{2}\right\|_{W^{1,2}(0, T ; Y)} \leq C\left\|u_{1}-u_{2}\right\|_{\mathcal{X}}
$$

To establish existence of solutions to Problem 4, we start with its equivalent form.

Problem 7 Find $u \in \mathcal{V}$ with $u^{\prime} \in \mathcal{W}$ and $\beta \in W^{1,2}(0, T ; Y)$ such that there exists $\xi \in \mathcal{X}^{*}$ and

$$
\left\{\begin{array}{l}
\int_{0}^{T} \quad\left(\left\langle u^{\prime \prime}(t), v(t)-u^{\prime}(t)\right\rangle+\left\langle A u^{\prime}(t)+B u(t)+M^{*} \xi(t), v(t)-u^{\prime}(t)\right\rangle\right. \\
\left.\quad+\varphi(v(t))-\varphi\left(u^{\prime}(t)\right)\right) d t \geq \int_{0}^{T}\left\langle f(t), v(t)-u^{\prime}(t)\right\rangle d t \quad \text { for all } v \in \mathcal{V}, \\
\xi(t) \in \partial J\left(\beta(t), M u^{\prime}(t)\right) \quad \text { for a.e. } t \in(0, T), \\
\beta^{\prime}(t)=F(t, M u(t), \beta(t)) \quad \text { for a.e. } t \in(0, T), \\
u(0)=u_{0}, \quad u^{\prime}(0)=v_{0}, \quad \beta(0)=\beta_{0} .
\end{array}\right.
$$

Proposition 8 Problems 4 and (7) are equivalent. 
Proof Let $(u, \beta, \xi)$ be a solution to Problem 4. Then, for any $v \in \mathcal{V}$,

$$
\begin{aligned}
& \left\langle u^{\prime \prime}(t)+A u^{\prime}(t)+B u(t)-f(t), v(t)-u^{\prime}(t)\right\rangle+\left\langle\xi(t), M\left(v(t)-u^{\prime}(t)\right)\right\rangle_{X^{*} \times X} \\
& +\varphi(v(t))-\varphi\left(u^{\prime}(t)\right) \geq 0 \text { for a.e. } t \in(0, T) .
\end{aligned}
$$

Integrating the above inequality on $(0, T)$, we see that the first inequality in (3.4) holds. Thus, $(u, \beta, \xi)$ is a solution to Problem 7 .

Conversely, assume $(u, \beta, \xi)$ is a solution of Problem 7 , and we need to show that the first inequality in (3.1) holds. Arguing by contradiction, we suppose that there exists a measurable subset $J \subset[0, T]$ with meas $(J)>0$ and $v^{*} \in V$ such that

$$
\begin{aligned}
& \left\langle u^{\prime \prime}(t)+A u^{\prime}(t)+B u(t)-f(t), v^{*}-u^{\prime}(t)\right\rangle+\left\langle\xi(t), M\left(v^{*}-u^{\prime}(t)\right)\right\rangle_{X^{*} \times X} \\
& +\varphi\left(v^{*}\right)-\varphi\left(u^{\prime}(t)\right)<0 \text { for all } t \in J .
\end{aligned}
$$

Since $u^{\prime} \in \mathcal{V}$, we define $\widehat{v} \in \mathcal{V}$ by

$$
\widehat{v}(t)= \begin{cases}v^{*}, & \text { if } t \in J, \\ u^{\prime}(t), & \text { otherwise. }\end{cases}
$$

Choose $v=\widehat{v}$ in the first inequality in (3.4) to obtain

$$
\begin{aligned}
0 \leq & \int_{0}^{T}\left\langle u^{\prime \prime}(t)+A u^{\prime}(t)+B u(t)-f(t), \widehat{v}(t)-u^{\prime}(t)\right\rangle d t \\
& +\int_{0}^{T}\left\langle\xi(t), M\left(\widehat{v}(t)-u^{\prime}(t)\right)\right\rangle_{X^{*} \times X} d t+\int_{0}^{T}\left(\varphi(\widehat{v}(t))-\varphi\left(u^{\prime}(t)\right)\right) d t \\
= & \int_{J}\left\langle u^{\prime \prime}(t)+A u^{\prime}(t)+B u(t)-f(t), v^{*}-u^{\prime}(t)\right\rangle d t \\
& +\int_{J}\left\langle\xi(t), M\left(v^{*}-u^{\prime}(t)\right)\right\rangle_{X^{*} \times X} d t+\int_{J}\left(\varphi\left(v^{*}\right)-\varphi\left(u^{\prime}(t)\right)\right) d t<0,
\end{aligned}
$$

which is a contradiction. Thus, $(u, \beta, \xi)$ is a solution to Problem 4.

The main idea in the existence proof is to carry out a mixed kind of temporally semi-discrete approximation and pass the limit as the time step approaches zero to find a solution to Problem 4. Let $N \in \mathbb{N}$ be a positive integer. We use a uniform partition of the time interval $[0, T]$ into $N$ equal length subintervals. The time step is $\tau=T / N$ and the partition points are $t_{k}=k \tau, 0 \leq k \leq N$. Define

$$
f_{\tau}^{k}=\frac{1}{\tau} \int_{t_{k-1}}^{t_{k}} f(t) d t \quad \text { for } k=1, \ldots, N .
$$

The semi-discrete scheme for Problem 4 is as follows:

Problem 9 Find $\left\{v_{\tau}^{k}\right\}_{k=0}^{N} \subset V,\left\{\xi_{\tau}^{k}\right\}_{k=1}^{N} \subset X^{*}$ and $\beta_{\tau} \in W^{1,2}(0, T ; Y)$ such that $v_{\tau}^{0}=v_{0}, u_{\tau}^{k}=u_{0}+$ $\tau \sum_{i=1}^{k} v_{\tau}^{i}, u_{\tau}^{0}=u_{0}, \beta_{\tau}(0)=\beta_{0}$, and for $1 \leq k \leq N$,

$$
\begin{gathered}
\left(\frac{v_{\tau}^{k}-v_{\tau}^{k-1}}{\tau}, v-v_{\tau}^{k}\right)_{H}+\left\langle A v_{\tau}^{k}+B u_{\tau}^{k}, v-v_{\tau}^{k}\right\rangle+\left\langle\xi_{\tau}^{k}, M\left(v-v_{\tau}^{k}\right)\right\rangle_{X^{*} \times X} \\
+\varphi(v)-\varphi\left(v_{\tau}^{k}\right) \geq\left\langle f_{\tau}^{k}, v-v_{\tau}^{k}\right\rangle \quad \text { for all } v \in V,
\end{gathered}
$$


with $\xi_{\tau}^{k} \in \partial J\left(\beta_{\tau}\left(t_{k-1}\right), M v_{\tau}^{k}\right)$ and

$$
\beta_{\tau}^{\prime}(t)=F\left(t, M \hat{u}_{\tau}(t), \beta_{\tau}(t)\right),
$$

where $\hat{u}_{\tau}$ is defined as follows:

$$
\hat{u}_{\tau}(t)= \begin{cases}\sum_{i=1}^{N} \chi_{\left(t_{i-1}, t_{i}\right]}(t) u_{\tau}^{i}, & t>0 \\ u_{0}, & t=0 .\end{cases}
$$

Here, $\chi_{\left(t_{i-1}, t_{i}\right]}$ denotes the characteristic function on the interval $\left(t_{i-1}, t_{i}\right]$, that is,

$$
\chi_{\left(t_{i-1}, t_{i}\right]}(t)= \begin{cases}1, & t \in\left(t_{i-1}, t_{i}\right] \\ 0, & \text { otherwise. }\end{cases}
$$

The following lemma shows that the hybrid iterative system formulated in Problem 9 is uniquely solvable.

Lemma 10 Assume that $H(A), H(B), H(J)(i)-(i i i), H(F), H(\varphi), H(M), H(f)$ and $H(0)$ (ii) are fulfilled. Then, there exists $\tau_{0}>0$ such that for all $\tau \in\left(0, \tau_{0}\right)$, Problem 9 has a unique solution.

Proof We prove the result by induction. Let $u_{\tau}^{0}, v_{\tau}^{0}, v_{\tau}^{1}, \ldots, v_{\tau}^{k-1}$ and $\beta_{\tau} \in W^{1,2}\left(0, t_{\tau}^{k-1} ; Y\right)$ be given. From (3.5), we have

$$
\begin{gathered}
\left(\frac{v_{\tau}^{k}-v_{\tau}^{k-1}}{\tau}, v-v_{\tau}^{k}\right)_{H}+\left\langle A v_{\tau}^{k}+\tau B v_{\tau}^{k}, v-v_{\tau}^{k}\right\rangle+\left\langle\xi_{\tau}^{k}, M\left(v-v_{\tau}^{k}\right)\right\rangle_{X^{*} \times X} \\
+\varphi(v)-\varphi\left(v_{\tau}^{k}\right) \geq\left\langle f_{\tau}^{k}-B u_{0}-\tau \sum_{i=1}^{k-1} B v_{\tau}^{i}, v-v_{\tau}^{k}\right\rangle \quad \text { for all } v \in V
\end{gathered}
$$

with $\xi_{\tau}^{k} \in \partial J\left(\beta_{\tau}\left(t_{k-1}\right), M v_{\tau}^{k}\right)$. In order to verify the solvability of the above inequality, it suffices to show that the following inclusion has at least one solution $w \in V$ :

$$
\frac{i^{*} i w}{\tau}+A w+\tau B w+M^{*} \partial J\left(\beta_{\tau}\left(t_{k-1}\right), M w\right)+\partial_{c} \varphi(w) \ni L_{\tau}^{k},
$$

where

$$
L_{\tau}^{k}=\frac{i^{*} i v_{\tau}^{k-1}}{\tau}+f_{\tau}^{k}-B u_{0}-\tau \sum_{i=1}^{k-1} B v_{\tau}^{i},
$$

$i$ denotes the embedding operator from $V$ to $H$, and the operator $\partial_{c} \varphi$ stands for the convex subdifferential of the function $\varphi$. We introduce the multivalued mappings $\mathcal{F}: D\left(\partial_{c} \varphi\right) \subset V \rightarrow 2^{V^{*}}$ and $\mathcal{G}: V \rightarrow 2^{V^{*}}$ by

$$
\begin{aligned}
& \mathcal{F}(w)=\partial_{c} \varphi(w) \quad \text { for all } w \in D\left(\partial_{c} \varphi\right), \\
& \mathcal{G}(w)=\frac{i^{*} i w}{\tau}+A w+\tau B w+M^{*} \partial J\left(\beta_{\tau}\left(t_{k-1}\right), M w\right) \quad \text { for all } w \in V .
\end{aligned}
$$

We will apply Theorem 3 and thus verify the conditions of the theorem.

Due to the convexity of $\varphi, \mathcal{F}$ is a maximal monotone operator ([10, Theorem 3.69]). 
To prove that $\mathcal{G}$ is a pseudomonotone operator, note that its boundedness follows from

$$
\begin{aligned}
\|\mathcal{G}(w)\|_{V^{*}} & \leq \frac{1}{\tau}\left\|i^{*} i w\right\|_{V^{*}}+\|A w\|_{V^{*}}+\tau\|B w\|_{V^{*}}+\left\|M^{*} \partial J\left(\beta_{\tau}\left(t_{k-1}\right), M w\right)\right\|_{V^{*}} \\
& \leq \frac{\|i\|_{\mathcal{L}(V, H)}^{2}}{\tau}\|w\|+a_{A}+b_{A}\|w\|+\tau\|B\|_{\mathcal{L}\left(V, V^{*}\right)}\|w\|+\|M\|_{\mathcal{L}(V, X)} c_{J}\left(1+\|M\|_{\mathcal{L}(V, X)}\|w\|\right) .
\end{aligned}
$$

We notice that the hypotheses $H(B)$ and the continuity of the embedding operator $i$ imply that the operator $\frac{i^{*} i}{\tau}+\tau B$ is bounded, continuous and monotone, hence it is also pseudomonotone, see [35, Theorem 3.69(ii)]. The growth condition on $\partial J$ (see $H(J)($ ii)) combined with $H(M)$ and Proposition 1 entail that $u \mapsto M^{*} \partial J\left(\beta_{\tau}\left(t_{k-1}\right), M u\right)$ is pseudomonotone. Therefore, since $A$ is pseudomonotone (see $H(A)(\mathrm{i})$ ), we conclude that $\mathcal{G}$ is a pseudomonotone operator, being a sum of pseudomonotone operators, see [35, Proposition 3.59(ii)].

Now, we show that the coercivity condition (2.1) is satisfied. Observe that

$$
\left\langle L_{\tau}^{k}, w-v_{0}\right\rangle \leq\left\|L_{\tau}^{k}\right\|_{V^{*}}\left(\|w\|+\left\|v_{0}\right\|\right) \leq C_{1}(\|w\|+1)
$$

for all $w \in V$, where $C_{1}=\left\|L_{\tau}^{k}\right\|_{V^{*}} \max \left\{1,\left\|v_{0}\right\|\right\}$. Let $w \in D\left(\partial_{c} \varphi\right), \xi \in \mathcal{F}(w)$ and $\eta \in \mathcal{G}(w)$. The assumptions on $\varphi$ guarantee that $\varphi$ is bounded below by an affine functional, see [1, Lemma 11.3.5]. Together with an application of the modified Cauchy-Schwarz inequality (2.2), we have the existence of constants $k_{1}, k_{2} \geq 0$ such that

$$
\left\langle\xi, w-v_{0}\right\rangle \geq \varphi(w)-\varphi\left(v_{0}\right) \geq-k_{1}-k_{2}\|w\|-\varphi\left(v_{0}\right) \geq-\varepsilon\|w\|^{2}-\frac{k_{2}^{2}}{4 \varepsilon}-k_{1}-\varphi\left(v_{0}\right)
$$

For any $\zeta \in \partial J\left(\beta_{\tau}\left(t_{k-1}\right), M(w)\right)$, condition $H(J)($ iii) implies

$$
\begin{aligned}
\left\langle\zeta, M\left(w-v_{0}\right)\right\rangle_{X^{*} \times X}=\langle\zeta & \left.-\zeta_{0}, M w\right\rangle_{X^{*} \times X}-\left\langle\zeta-\zeta_{0}, M v_{0}\right\rangle_{X^{*} \times X}+\left\langle\zeta_{0}, M\left(w-v_{0}\right)\right\rangle_{X^{*} \times X} \\
\geq & -m_{J}\|M\|_{\mathcal{L}(V, X)}^{2}\|w\|^{2}-\left(\|\zeta\|_{X^{*}}+\left\|\zeta_{0}\right\|_{X^{*}}\right)\|M\|_{\mathcal{L}(V, X)}\left\|v_{0}\right\| \\
& +\left\|\zeta_{0}\right\|_{X^{*}}\|M\|_{\mathcal{L}(V, X)}\left(\|w\|+\left\|v_{0}\right\|\right)
\end{aligned}
$$

for all $\zeta_{0} \in \partial J\left(M 0_{V}\right)$, where by hypothesis $H(J)(\mathrm{ii}),\|\zeta\|_{X^{*}} \leq c_{J}\left(1+\|M\|_{\mathcal{L}(V, X)}\|w\|\right)$. We use these inequalities and apply the modified Cauchy-Schwarz inequality (2.2) to obtain

$$
\left\langle\zeta, M\left(w-v_{0}\right)\right\rangle_{X^{*} \times X} \geq-m_{J}\|M\|_{\mathcal{L}(V, X)}^{2}\|w\|^{2}-\varepsilon\|w\|^{2}-c_{1}(\varepsilon)
$$

where the constant $c_{1}(\varepsilon)>0$ is independent of $w$. Similarly, it follows from hypotheses $H(A)(\mathrm{ii})-$ (iii), $H(B)$ that for a constant $c_{2}(\varepsilon)>0$,

$$
\begin{aligned}
\langle A w, & \left.w-v_{0}\right\rangle+\tau\left\langle B w, w-v_{0}\right\rangle \\
& =\left\langle A w-A 0_{V}, w\right\rangle+\left\langle A 0_{V}, w\right\rangle-\left\langle A w, v_{0}\right\rangle+\tau\langle B w, w\rangle-\tau\left\langle B w, v_{0}\right\rangle \\
& \geq m_{A}\|w\|^{2}-c_{A}\|w\|_{H}^{2}+\tau \varrho_{B}\|w\|^{2}-\left\|A 0_{V}\right\|_{V^{*}}\|w\|-\left(a_{A}+b_{A}\|w\|\right)\left\|v_{0}\right\|-\tau\|B\|\|w\|\left\|v_{0}\right\| \\
& \geq\left(m_{A}+\tau \varrho_{B}-\varepsilon\right)\|w\|^{2}-c_{A}\|w\|_{H}^{2}-c_{2}(\varepsilon)
\end{aligned}
$$


So, taking into account (3.9)-(3.11), we have

$$
\begin{aligned}
\left\langle\xi+\eta, w-v_{0}\right\rangle= & \frac{1}{\tau}\left(w, w-v_{0}\right)_{H}+\left\langle A w, w-v_{0}\right\rangle+\tau\left\langle B w, w-v_{0}\right\rangle \\
& +\left\langle\eta, w-v_{0}\right\rangle+\left\langle\zeta, M\left(w-v_{0}\right)\right\rangle_{X^{*} \times X} \\
\geq & \frac{1}{2 \tau}\|w\|_{H}^{2}-\frac{1}{2 \tau}\left\|v_{0}\right\|_{H}^{2}+\left(m_{A}+\tau \varrho_{B}-\varepsilon\right)\|w\|^{2}-c_{A}\|w\|_{H}^{2}-c_{2}(\varepsilon) \\
& -\varepsilon\|w\|^{2}-\frac{k_{2}^{2}}{4 \varepsilon}-k_{1}-\varphi\left(v_{0}\right)-m_{J}\|M\|_{\mathcal{L}(V, X)}^{2}\|w\|^{2}-\varepsilon\|w\|^{2}-c_{1}(\varepsilon) .
\end{aligned}
$$

Combining (3.8) and (3.12), after some elementary manipulations, we find that

$$
\left\langle\xi+\eta-L_{\tau}^{k}, w-v_{0}\right\rangle \geq\left(\frac{1}{2 \tau}-c_{A}\right)\|w\|_{H}^{2}+\left(m_{A}-m_{J}\|M\|_{\mathcal{L}(V, X)}^{2}-3 \varepsilon\right)\|w\|^{2}-C_{1}\|w\|+c_{3}(\varepsilon) .
$$

Taking $\tau_{0}=1 /\left(2 c_{A}\right)$ and $\varepsilon=\left(m_{A}-m_{J}\|M\|_{\mathcal{L}(V, X)}^{2}\right) / 6$, we see easily from (3.13) that if $\|w\|$ is sufficiently large, then the coercivity condition (2.1) is valid.

We now apply Theorem 3 to conclude the existence of an element $v_{\tau}^{k} \in V$ solving the problem (3.7), which is a solution of the variational-hemivariational inequality (3.5).

The uniqueness of a solution of the inequality (3.5) is proved by a standard approach. Assume $v_{\tau}^{k}$ and $\widetilde{v}_{\tau}^{k}$ are two solutions of the problem (3.5). Then for all $v \in V$,

$$
\begin{aligned}
& \left(\frac{v_{\tau}^{k}-v_{\tau}^{k-1}}{\tau}, v-v_{\tau}^{k}\right)_{H}+\left\langle A v_{\tau}^{k}+B u_{\tau}^{k}, v-v_{\tau}^{k}\right\rangle+\left\langle\xi_{\tau}^{k}, M\left(v-v_{\tau}^{k}\right)\right\rangle_{X^{*} \times X}+\varphi(v) \\
& \quad-\varphi\left(v_{\tau}^{k}\right) \geq\left\langle f_{\tau}^{k}, v-v_{\tau}^{k}\right\rangle, \\
& \left(\frac{\widetilde{v}_{\tau}^{k}-v_{\tau}^{k-1}}{\tau}, v-\widetilde{v}_{\tau}^{k}\right)_{H}+\left\langle A \widetilde{v}_{\tau}^{k}+B \widetilde{u}_{\tau}^{k}, v-\widetilde{v}_{\tau}^{k}\right\rangle+\left\langle\widetilde{\xi}_{\tau}^{k}, M\left(v-\widetilde{v}_{\tau}^{k}\right)\right\rangle_{X^{*} \times X}+\varphi(v) \\
& \quad-\varphi\left(\widetilde{v}_{\tau}^{k}\right) \geq\left\langle f_{\tau}^{k}, v-\widetilde{v}_{\tau}^{k}\right\rangle,
\end{aligned}
$$

where $\xi_{\tau}^{k} \in \partial J\left(\beta\left(t_{k-1}\right), M v_{\tau}^{k}\right)$ and $\widetilde{\xi}_{\tau}^{k} \in \partial J\left(\beta\left(t_{k-1}\right), M \widetilde{v}_{\tau}^{k}\right)$. Taking $v=\widetilde{v}_{\tau}^{k}$ in the first inequality and $v=v_{\tau}^{k}$ in the second one, we add the two resulting inequalities to get

$$
\begin{aligned}
& \left(\frac{v_{\tau}^{k}-\widetilde{v}_{\tau}^{k}}{\tau}, v_{\tau}^{k}-\widetilde{v}_{\tau}^{k}\right)_{H}+\left\langle A v_{\tau}^{k}-A \widetilde{v}_{\tau}^{k}, v_{\tau}^{k}-\widetilde{v}_{\tau}^{k}\right\rangle+\left\langle B u_{\tau}^{k}-B \widetilde{u}_{\tau}^{k}, v_{\tau}^{k}-\widetilde{v}_{\tau}^{k}\right\rangle \\
& \quad+\left\langle\xi_{\tau}^{k}-\widetilde{\xi}_{\tau}^{k}, M\left(v_{\tau}^{k}-\widetilde{v}_{\tau}^{k}\right)\right\rangle_{X^{*} \times X} \leq 0 .
\end{aligned}
$$

Keeping in mind hypotheses $H(A)($ iii), $H(B)$ and $H(J)($ iii), we have

$$
\left(\frac{1}{\tau}-c_{A}\right)\left\|v_{\tau}^{k}-\widetilde{v}_{\tau}^{k}\right\|_{H}^{2}+\left(m_{A}-m_{J}\|M\|_{\mathcal{L}(V, X)}^{2}\right)\left\|v_{\tau}^{k}-\widetilde{v}_{\tau}^{k}\right\|^{2}+\tau \varrho_{B}\left\|v_{\tau}^{k}-\widetilde{v}_{\tau}^{k}\right\|^{2} \leq 0 .
$$

Since $\tau<\tau_{0}=\frac{1}{2 c_{A}}$, the smallness condition $m_{A}>m_{J}\|M\|_{\mathcal{L}(V, X)}^{2}$ guarantees that $v_{\tau}^{k}=\widetilde{v}_{\tau}^{k}$. This completes the proof of uniqueness.

To finish the proof, it remains to show that there exists a unique solution to the following Cauchy problem

$$
\left\{\begin{array}{l}
\beta_{\tau}^{\prime}(t)=F\left(t, \hat{u}_{\tau}(t), \beta_{\tau}(t)\right) \quad \text { for a.e. } t \in\left[0, t_{k}\right] \\
\beta_{\tau}(0)=\beta_{0}
\end{array}\right.
$$


Since $u_{\tau}^{i}=u_{0}+\tau \sum_{j=1}^{i} v_{\tau}^{j}$ for $i=1,2, \ldots, k$, it is easy to obtain $u_{\tau}^{1}, u_{\tau}^{2}, \ldots, u_{\tau}^{k}$. Therefore, the function $\hat{u}_{\tau}(t)=\sum_{i=1}^{k} \chi_{\left(t_{i-1}, t_{i}\right]}(t) u_{\tau}^{i}$ with $\hat{u}_{\tau}(0)=u_{0}$ is well defined on $\left[0, t_{k}\right]$ and $\hat{u}_{\tau} \in L^{2}\left(0, t_{k} ; V\right)$. Lemma 6 ensures that the above Cauchy problem has a unique solution $\beta_{\tau} \in W^{1,2}\left(0, t_{k} ; Y\right)$.

Denote $z_{\tau}^{k}=\frac{v_{\tau}^{k}-v_{\tau}^{k-1}}{\tau}$ for $k=1,2, \ldots, N$ and $z_{\tau}^{0}=z_{0}$, where $z_{0}$ is given in hypothesis $H(0)(\mathrm{i})$. The following lemma provides a priori bounds for the solution of Problem 9.

Lemma 11 Assume $H(A), H(B), H(J)(i)-(i i i), H(M), H(F), H(\varphi), H(0)$ and $H(f)$. Then, there exists $\tau_{0}>0$ and a constant $C>0$ independent of $\tau$ such that for all $\tau \in\left(0, \tau_{0}\right)$ :

$$
\begin{aligned}
& \max _{1 \leq k \leq N}\left\|u_{\tau}^{k}\right\| \leq C, \\
& \max _{1 \leq k \leq N}\left\|v_{\tau}^{k}\right\| \leq C, \\
& \max _{1 \leq k \leq N}\left\|z_{\tau}^{k}\right\|_{H} \leq C, \\
& \max _{1 \leq k \leq N}\left\|\xi_{\tau}^{k}\right\|_{X^{*}} \leq C, \\
& \sum_{k=1}^{N}\left\|v_{\tau}^{k}-v_{\tau}^{k-1}\right\|^{2} \leq C, \\
& \sum_{k=1}^{N}\left\|z_{\tau}^{k}-z_{\tau}^{k-1}\right\|_{H}^{2} \leq C, \\
& \tau \sum_{k=1}^{N}\left\|z_{\tau}^{k}\right\|^{2} \leq C .
\end{aligned}
$$

Proof For $k \geq 2$, we let $v=v_{\tau}^{k-1}$ in (3.5) for $k$ and let $v=v_{\tau}^{k}$ in (3.5) with $k$ replaced by $k-1$ to get

$$
\begin{aligned}
& \left(\frac{v_{\tau}^{k}-v_{\tau}^{k-1}}{\tau}, v_{\tau}^{k-1}-v_{\tau}^{k}\right)_{H}+\left\langle A v_{\tau}^{k}+B u_{\tau}^{k}, v_{\tau}^{k-1}-v_{\tau}^{k}\right\rangle+\left\langle\xi_{\tau}^{k}, M\left(v_{\tau}^{k-1}-v_{\tau}^{k}\right)\right\rangle_{X^{*} \times X} \\
& \quad+\varphi\left(v_{\tau}^{k-1}\right)-\varphi\left(v_{\tau}^{k}\right) \geq\left\langle f_{\tau}^{k}, v_{\tau}^{k-1}-v_{\tau}^{k}\right\rangle, \\
& \left(\frac{v_{\tau}^{k-1}-v_{\tau}^{k-2}}{\tau}, v_{\tau}^{k}-v_{\tau}^{k-1}\right)_{H}+\left\langle A v_{\tau}^{k-1}+B u_{\tau}^{k-1}, v_{\tau}^{k}-v_{\tau}^{k-1}\right\rangle+\varphi\left(v_{\tau}^{k}\right)-\varphi\left(v_{\tau}^{k-1}\right) \\
& \quad+\left\langle\xi_{\tau}^{k-1}, M\left(v_{\tau}^{k}-v_{\tau}^{k-1}\right)\right\rangle_{X^{*} \times X} \geq\left\langle f_{\tau}^{k-1}, v_{\tau}^{k}-v_{\tau}^{k-1}\right\rangle,
\end{aligned}
$$

where $\xi_{\tau}^{k} \in \partial J\left(\beta_{\tau}\left(t_{k-1}\right), M v_{\tau}^{k}\right)$ and $\xi_{\tau}^{k-1} \in \partial J\left(\beta_{\tau}\left(t_{k-2}\right), M v_{\tau}^{k-1}\right)$. Add the above two inequalities,

$$
\begin{gathered}
\left(\frac{v_{\tau}^{k}-v_{\tau}^{k-1}-\left(v_{\tau}^{k-1}-v_{\tau}^{k-2}\right)}{\tau}, v_{\tau}^{k}-v_{\tau}^{k-1}\right)_{H}+\left\langle A v_{\tau}^{k}-A v_{\tau}^{k-1}, v_{\tau}^{k}-v_{\tau}^{k-1}\right\rangle \\
+\left\langle B u_{\tau}^{k}-B u_{\tau}^{k-1}, v_{\tau}^{k}-v_{\tau}^{k-1}\right\rangle+\left\langle\xi_{\tau}^{k}-\xi_{\tau}^{k-1}, M\left(v_{\tau}^{k}-v_{\tau}^{k-1}\right)\right\rangle_{X^{*} \times X} \\
\leq\left\langle f_{\tau}^{k}-f_{\tau}^{k-1}, v_{\tau}^{k}-v_{\tau}^{k-1}\right\rangle .
\end{gathered}
$$

Making use of the symbol $z_{\tau}^{k}=\frac{v_{\tau}^{k}-v_{\tau}^{k-1}}{\tau}$ and noting that $u_{\tau}^{k}-u_{\tau}^{k-1}=\tau v_{\tau}^{k}$, we rewrite the above inequality as 


$$
\begin{aligned}
\left(z_{\tau}^{k}-\right. & \left.z_{\tau}^{k-1}, z_{\tau}^{k}\right)_{H}+\frac{1}{\tau}\left\langle A v_{\tau}^{k}-A v_{\tau}^{k-1}, v_{\tau}^{k}-v_{\tau}^{k-1}\right\rangle+\left\langle B v_{\tau}^{k}, v_{\tau}^{k}-v_{\tau}^{k-1}\right\rangle \\
& +\frac{1}{\tau}\left\langle\xi_{\tau}^{k}-\xi_{\tau}^{k-1}, M\left(v_{\tau}^{k}-v_{\tau}^{k-1}\right)\right\rangle_{X^{*} \times X} \leq \frac{1}{\tau}\left\langle f_{\tau}^{k}-f_{\tau}^{k-1}, v_{\tau}^{k}-v_{\tau}^{k-1}\right\rangle .
\end{aligned}
$$

Exploiting the identities

$$
\begin{aligned}
& \left(z_{\tau}^{k}-z_{\tau}^{k-1}, z_{\tau}^{k}\right)_{H}=\frac{1}{2}\left\|z_{\tau}^{k}\right\|_{H}^{2}-\frac{1}{2}\left\|z_{\tau}^{k-1}\right\|_{H}^{2}+\frac{1}{2}\left\|z_{\tau}^{k}-z_{\tau}^{k-1}\right\|_{H}^{2}, \\
& \left\langle B v_{\tau}^{k}, v_{\tau}^{k}-v_{\tau}^{k-1}\right\rangle=\frac{1}{2}\left(\left\langle B v_{\tau}^{k}, v_{\tau}^{k}\right\rangle-\left\langle B v_{\tau}^{k-1}, v_{\tau}^{k-1}\right\rangle+\left\langle B\left(v_{\tau}^{k}-v_{\tau}^{k-1}\right), v_{\tau}^{k}-v_{\tau}^{k-1}\right\rangle\right),
\end{aligned}
$$

and assumptions $H(A)(\mathrm{iii}), H(B)$ and $H(J)(\mathrm{iii})$, we deduce that

$$
\begin{aligned}
\frac{1}{2}\left\|z_{\tau}^{k}\right\|_{H}^{2}-\frac{1}{2}\left\|z_{\tau}^{k-1}\right\|_{H}^{2}+\frac{1}{2}\left\|z_{\tau}^{k}-z_{\tau}^{k-1}\right\|_{H}^{2}+\frac{m_{A}}{\tau}\left\|v_{\tau}^{k}-v_{\tau}^{k-1}\right\|^{2}-\frac{c_{A}}{\tau}\left\|v_{\tau}^{k}-v_{\tau}^{k-1}\right\|_{H}^{2} \\
+\frac{1}{2}\left\|v_{\tau}^{k}\right\|_{B}^{2}-\frac{1}{2}\left\|v_{\tau}^{k-1}\right\|_{B}^{2}+\frac{1}{2}\left\|v_{\tau}^{k}-v_{\tau}^{k-1}\right\|_{B}^{2}-\frac{m_{J}}{\tau}\left\|M\left(v_{\tau}^{k}-v_{\tau}^{k-1}\right)\right\|_{X}^{2} \\
-\frac{m_{J}}{\tau}\left\|\beta_{\tau}\left(t_{k-1}\right)-\beta_{\tau}\left(t_{k-2}\right)\right\|_{Y}\left\|M\left(v_{\tau}^{k}-v_{\tau}^{k-1}\right)\right\|_{X} \leq \frac{1}{\tau}\left\langle f_{\tau}^{k}-f_{\tau}^{k-1}, v_{\tau}^{k}-v_{\tau}^{k-1}\right\rangle,
\end{aligned}
$$

where $\|v\|_{B}:=\langle B v, v\rangle$ for $v \in V$. Now by $H(F)$,

$$
\left\|\beta_{\tau}\left(t_{k-1}\right)-\beta_{\tau}\left(t_{k-2}\right)\right\|_{Y} \leq \int_{t_{k-2}}^{t_{k-1}}\left\|F\left(s, u_{\tau}^{k-1}, \beta_{\tau}(s)\right)\right\|_{Y} d s \leq M_{F} \tau .
$$

Also,

$$
\frac{1}{\tau}\left\langle f_{\tau}^{k}-f_{\tau}^{k-1}, v_{\tau}^{k}-v_{\tau}^{k-1}\right\rangle \leq \varepsilon \tau\left\|z_{\tau}^{k}\right\|^{2}+\frac{C}{\tau}\left\|f_{\tau}^{k}-f_{\tau}^{k-1}\right\|_{V^{*}}^{2} .
$$

Hence, from (3.21), we have

$$
\begin{gathered}
\frac{1}{2}\left\|z_{\tau}^{k}\right\|_{H}^{2}-\frac{1}{2}\left\|z_{\tau}^{k-1}\right\|_{H}^{2}+\frac{1}{2}\left\|z_{\tau}^{k}-z_{\tau}^{k-1}\right\|_{H}^{2}+\frac{1}{2}\left\|v_{\tau}^{k}\right\|_{B}^{2}-\frac{1}{2}\left\|v_{\tau}^{k-1}\right\|_{B}^{2} \\
+\frac{1}{2}\left\|v_{\tau}^{k}-v_{\tau}^{k-1}\right\|_{B}^{2}+\tau\left(m_{A}-m_{J}\|M\|_{\mathcal{L}(V, X)}^{2}-2 \varepsilon\right)\left\|z_{\tau}^{k}\right\|^{2} \\
\leq \frac{C}{\tau}\left\|f_{\tau}^{k}-f_{\tau}^{k-1}\right\|_{V^{*}}^{2}+c_{A} \tau\left\|z_{\tau}^{k}\right\|_{H}^{2}+\frac{m_{J}^{2}\|M\|_{\mathcal{L}(V, X)}^{2} M_{F}^{2} \tau}{4 \varepsilon} .
\end{gathered}
$$

Adding this inequality from $k=1$ to $k=n$, where $1 \leq n \leq N$, and using the coercivity of operator $B$, we deduce that

$$
\begin{gathered}
\frac{1}{2}\left\|z_{\tau}^{n}\right\|_{H}^{2}-\frac{1}{2}\left\|z_{\tau}^{0}\right\|_{H}^{2}+\frac{1}{2} \sum_{k=1}^{n}\left\|z_{\tau}^{k}-z_{\tau}^{k-1}\right\|_{H}^{2}+\frac{\varrho_{B}}{2}\left\|v_{\tau}^{n}\right\|^{2}-\frac{1}{2}\left\|v_{\tau}^{0}\right\|_{B}^{2} \\
+\frac{\varrho_{B}}{2} \sum_{k=1}^{n}\left\|v_{\tau}^{k}-v_{\tau}^{k-1}\right\|^{2}+\tau\left(m_{A}-m_{J}\|M\|_{\mathcal{L}(V, X)}^{2}-2 \varepsilon\right) \sum_{k=1}^{n}\left\|z_{\tau}^{k}\right\|^{2} \\
\leq \frac{C}{\tau} \sum_{k=1}^{n}\left\|f_{\tau}^{k}-f_{\tau}^{k-1}\right\|_{V^{*}}^{2}+c_{A} \tau \sum_{k=1}^{n}\left\|z_{\tau}^{k}\right\|_{H}^{2}+C .
\end{gathered}
$$


Now

$$
\begin{aligned}
& f_{\tau}^{k}-f_{\tau}^{k-1}=\frac{1}{\tau} \int_{t_{k-1}}^{t_{k}}[f(s)-f(s-\tau)] d s=\frac{1}{\tau} \int_{t_{k-1}}^{t_{k}} \int_{s-\tau}^{s} f^{\prime}\left(s_{1}\right) d s_{1} d s, \\
& \left\|f_{\tau}^{k}-f_{\tau}^{k-1}\right\|_{V^{*}} \leq \int_{t_{k-1}}^{t_{k}}\left\|f^{\prime}(s)\right\|_{V^{*}} d s,
\end{aligned}
$$

and so

$$
\frac{C}{\tau} \sum_{k=1}^{n}\left\|f_{\tau}^{k}-f_{\tau}^{k-1}\right\|_{V^{*}}^{2} \leq C\|f\|_{H^{1}\left(0, T ; V^{*}\right)}^{2}
$$

Thus, from (3.22) with $\varepsilon=\left(m_{A}-m_{J}\|M\|_{\mathcal{L}(V, X)}^{2}\right) / 4$, we find that for some constant $C>0$ depending on the data but independent of $\tau$ such that

$$
\begin{aligned}
\left\|z_{\tau}^{n}\right\|_{H}^{2} & +\sum_{k=1}^{n}\left\|z_{\tau}^{k}-z_{\tau}^{k-1}\right\|_{H}^{2}+\left\|v_{\tau}^{n}\right\|^{2}+\sum_{k=1}^{n}\left\|v_{\tau}^{k}-v_{\tau}^{k-1}\right\|^{2}+\tau \sum_{k=1}^{n}\left\|z_{\tau}^{k}\right\|^{2} \\
\leq & =C \tau \sum_{k=1}^{n}\left\|z_{\tau}^{k}\right\|_{H}^{2} .
\end{aligned}
$$

By a discrete version of the Gronwall's inequality, see [22, Lemma 7.26], we deduce from (3.24) that if $\tau$ is sufficiently small:

$$
\max _{1 \leq n \leq N}\left\|z_{\tau}^{n}\right\|_{H}^{2}+\sum_{k=1}^{N}\left\|z_{\tau}^{k}-z_{\tau}^{k-1}\right\|_{H}^{2}+\max _{1 \leq n \leq N}\left\|v_{\tau}^{n}\right\|^{2}+\sum_{k=1}^{N}\left\|v_{\tau}^{k}-v_{\tau}^{k-1}\right\|^{2}+\tau \sum_{k=1}^{N}\left\|z_{\tau}^{k}\right\|^{2} \leq C .
$$

Therefore, (3.15), (3.16) and (3.18)-(3.20) are valid. The bound (3.14) can be obtained immediately from $u_{\tau}^{k}=u_{0}+\tau \sum_{i=1}^{k} v_{\tau}^{i}$ and (3.15). By hypotheses $H(M), H(J)(\mathrm{ii})$ and (3.15), we have

$$
\left\|\xi_{\tau}^{k}\right\|_{X^{*}} \leq c_{J}\left(1+\left\|M v_{\tau}^{k}\right\|_{X}\right) \leq C,
$$

that is, (3.17) holds. This completes the proof of the lemma.

For a fixed $\tau>0$, we define piecewise affine functions $u_{\tau}, v_{\tau}, z_{\tau}$ and piecewise constant interpolant functions $\bar{u}_{\tau}, \bar{v}_{\tau}, \bar{z}_{\tau}, f_{\tau}, \xi_{\tau}$ by

$$
\begin{aligned}
& u_{\tau}(t)=u_{\tau}^{k}+\frac{t-t_{k}}{\tau}\left(u_{\tau}^{k}-u_{\tau}^{k-1}\right) \quad \text { for } t \in\left(t_{k-1}, t_{k}\right] \text {, } \\
& v_{\tau}(t)=v_{\tau}^{k}+\frac{t-t_{k}}{\tau}\left(v_{\tau}^{k}-v_{\tau}^{k-1}\right) \quad \text { for } t \in\left(t_{k-1}, t_{k}\right] \text {, } \\
& z_{\tau}(t)=z_{\tau}^{k}+\frac{t-t_{k}}{\tau}\left(z_{\tau}^{k}-z_{\tau}^{k-1}\right) \quad \text { for } t \in\left(t_{k-1}, t_{k}\right], \\
& \bar{u}_{\tau}(t)= \begin{cases}u_{\tau}^{k}, & t \in\left(t_{k-1}, t_{k}\right], \\
u_{\tau}^{0}, & t=0,\end{cases} \\
& \bar{v}_{\tau}(t)= \begin{cases}v_{\tau}^{k}, & t \in\left(t_{k-1}, t_{k}\right], \\
v_{\tau}^{0}, & t=0,\end{cases} \\
& \bar{z}_{\tau}(t)= \begin{cases}z_{\tau}^{k}, & t \in\left(t_{k-1}, t_{k}\right], \\
z_{\tau}^{0}, & t=0,\end{cases}
\end{aligned}
$$




$$
\begin{aligned}
& f_{\tau}(t)= \begin{cases}f_{\tau}^{k}, & t \in\left(t_{k-1}, t_{k}\right], \\
f(0), & t=0,\end{cases} \\
& \xi_{\tau}(t)= \begin{cases}\xi_{\tau}^{k}, & t \in\left(t_{k-1}, t_{k}\right], \\
\xi_{0}, & t=0 .\end{cases}
\end{aligned}
$$

For these functions, based on Lemma 11, we present the following estimates.

Lemma 12 Under assumptions $H(A), H(B), H(J)(i)-(i i i), H(M), H(F), H(\varphi), H(0)$ and $H(f)$, there exist $\tau_{0}>0$ and $C>0$ independent of $\tau$, such that for all $\tau \in\left(0, \tau_{0}\right)$, we have

$$
\begin{aligned}
& \left\|\bar{u}_{\tau}\right\|_{L^{\infty}(0, T ; V)} \leq C, \\
& \left\|\bar{v}_{\tau}\right\|_{L^{\infty}(0, T ; V)} \leq C, \\
& \left\|\bar{z}_{\tau}\right\|_{L^{\infty}(0, T ; H)} \leq C, \\
& \left\|\xi_{\tau}\right\|_{\mathcal{X}^{*}} \leq C, \\
& \left\|u_{\tau}\right\|_{C(0, T ; V)} \leq C, \\
& \left\|v_{\tau}\right\|_{C(0, T ; V)} \leq C, \\
& \left\|z_{\tau}\right\|_{C(0, T ; H)} \leq C, \\
& \left\|\bar{u}_{\tau}\right\|_{M^{2,2}\left(0, T ; V, V^{*}\right)} \leq C, \\
& \left\|\bar{v}_{\tau}\right\|_{M^{2,2}\left(0, T ; V, V^{*}\right)} \leq C .
\end{aligned}
$$

Proof The inequalities (3.14)-(3.17) imply (3.25)-(3.31). The bound (3.25) shows that $\left\{\bar{u}_{\tau}\right\}$ is bounded in $\mathcal{V}$. Without loss of generality, we may consider a division $0=a_{0}<a_{1}<\cdots<a_{n}=T$ with $a_{i} \in\left(\left(m_{i}-1\right) \tau, m_{i} \tau\right]$. Then, one has $\bar{u}_{\tau}\left(a_{i}\right)=u_{\tau}^{m_{i}}$ with $m_{0}=0, m_{n}=N$ and $m_{i+1}>m_{i}$ for $i=1,2, \ldots, N-1$. Using the continuity of the embedding $V \subset V^{*}$, we deduce

$$
\begin{aligned}
\left\|\bar{u}_{\tau}\right\|_{B V^{2}\left(0, T ; V^{*}\right)}= & \sum_{i=1}^{n}\left\|u_{\tau}^{m_{i}}-u_{\tau}^{m_{i}-1}\right\|_{V^{*}}^{2} \leq \sum_{i=1}^{n}\left(\left(m_{i}-m_{i-1}\right) \sum_{k=m_{i-1}+1}^{m_{i}}\left\|u_{\tau}^{k}-u_{\tau}^{k-1}\right\|_{V^{*}}^{2}\right) \\
& \leq\left(\sum_{i=1}^{n}\left(m_{i}-m_{i-1}\right)\right)\left(\sum_{i=1}^{n} \sum_{k=m_{i-1}+1}^{m_{i}}\left\|u_{\tau}^{k}-u_{\tau}^{k-1}\right\|_{V^{*}}^{2}\right)=N \sum_{k=1}^{N}\left\|u_{\tau}^{k}-u_{\tau}^{k-1}\right\|_{V^{*}}^{2} \\
= & T \tau \sum_{k=1}^{N}\left\|\frac{u_{\tau}^{k}-u_{\tau}^{k-1}}{\tau}\right\|_{V^{*}}^{2}=T \tau \sum_{k=1}^{N}\left\|v_{\tau}^{k}\right\|_{V^{*}}^{2} \leq C T \tau \sum_{k=1}^{N}\left\|v_{\tau}^{k}\right\|^{2} .
\end{aligned}
$$

Obviously, we have $\left\|\bar{u}_{\tau}\right\|_{B V^{2}\left(0, T ; V^{*}\right)} \leq C$ due to (3.15). Therefore, (3.32) is valid.

Analogously, by (3.26), $\left\{\bar{v}_{\tau}\right\}$ is bounded in $\mathcal{V}$. Similar to the derivation of (3.34), we have

$$
\left\|\bar{v}_{\tau}\right\|_{B V^{2}\left(0, T ; V^{*}\right)} \leq C T \tau \sum_{k=1}^{N}\left\|z_{\tau}^{k}\right\|^{2},
$$

which is bounded by (3.20). Thus, the bound (3.33) holds.

We are now ready to show the existence of solution for Problem 4. 
Theorem 13 Assume $H(A), H(B), H(J), H(M), H(F), H(\varphi), H(f)$ and $H(0)$. Consider a sequence $\{\tau\}$ converging to zero. Then, for a subsequence, still denoted by $\{\tau\}$, we have

$$
\begin{aligned}
& \bar{u}_{\tau} \rightarrow u \text { weakly in } \mathcal{V}, \\
& u_{\tau} \rightarrow u \text { weakly in } \mathcal{V}, \\
& \bar{v}_{\tau} \rightarrow u^{\prime} \text { weakly in } \mathcal{V}, \\
& v_{\tau} \rightarrow u^{\prime} \text { weakly in } \mathcal{V}, \\
& \mathcal{M}\left(\bar{v}_{\tau}\right) \rightarrow \mathcal{M}\left(u^{\prime}\right) \text { in } \mathcal{X}, \\
& \xi_{\tau} \rightarrow \xi \text { weakly in } \mathcal{X}^{*}, \\
& \bar{z}_{\tau} \rightarrow u^{\prime \prime} \text { weakly in } \mathcal{H}, \\
& \beta_{\tau} \rightarrow \beta \text { in } W^{1,2}(0, T ; Y), \\
& \bar{v}_{\tau} \rightarrow u^{\prime} \text { in } \mathcal{H},
\end{aligned}
$$

for some $(u, \xi, \beta) \in \mathcal{V} \times \mathcal{X}^{*} \times W^{1,2}(0, T ; Y)$ with $u^{\prime} \in \mathcal{W}$. Moreover, the limit $(u, \beta)$ is a solution to Problem 4, $\xi$ being a corresponding element of the subdifferential.

Proof It follows from (3.25) and the reflexivity of $\mathcal{V}$ that there exists a function $u \in \mathcal{V}$ such that (3.35) holds. Note that

$$
\left\|u_{\tau}-\bar{u}_{\tau}\right\|_{\mathcal{V}}^{2}=\sum_{k=1}^{N} \int_{t_{k-1}}^{t_{k}} \frac{\left(t-t_{k}\right)^{2}}{\tau^{2}}\left\|u_{\tau}^{k}-u_{\tau}^{k-1}\right\|^{2} d t=\frac{\tau^{3}}{3} \sum_{k=1}^{N}\left\|v_{\tau}^{k}\right\|^{2} .
$$

From the boundedness of $\tau \sum_{k=1}^{N}\left\|v_{\tau}^{k}\right\|^{2}$ (see (3.15)), it follows that $u_{\tau} \rightarrow u$ weakly in $\mathcal{V}$ as well, that is, (3.36) holds. By (3.26), there exists $v \in \mathcal{V}$ such that $\bar{v}_{\tau} \rightarrow v$ weakly in $\mathcal{V}$. Because of $u_{\tau}^{\prime}=\bar{v}_{\tau}$, we readily get $v=u^{\prime}$, so the convergence result (3.37) is obtained. In addition,

$$
\left\|v_{\tau}-\bar{v}_{\tau}\right\|_{\mathcal{V}}^{2}=\sum_{k=1}^{N} \int_{t_{k-1}}^{t_{k}} \frac{\left(t-t_{k}\right)^{2}}{\tau^{2}}\left\|v_{\tau}^{k}-v_{\tau}^{k-1}\right\|^{2} d t=\frac{\tau}{3} \sum_{k=1}^{N}\left\|v_{\tau}^{k}-v_{\tau}^{k-1}\right\|^{2}
$$

which by the boundedness of $\sum_{k=1}^{N}\left\|v_{\tau}^{k}-v_{\tau}^{k-1}\right\|^{2}$ (see (3.18)) implies that $v_{\tau} \rightarrow u^{\prime}$ weakly in $\mathcal{V}$, that is, (3.38) is valid.

The assumption $H(M)$ together with (3.33) and (3.37) ensures that (3.39) is true. Moreover, the boundedness of $\left\{\xi_{\tau}\right\}$ (see (3.28)) and the reflexivity of $\mathcal{X}^{*}$ entail that there exists $\xi \in \mathcal{X}^{*}$ such that (3.40) is satisfied. Similarly, since $\left\{\bar{z}_{\tau}\right\}$ is bounded in $\mathcal{H}$ (see (3.27)), there exists $w \in \mathcal{H}$ such that $\bar{z}_{\tau} \rightarrow w$ weakly in $\mathcal{H}$. Hence, exploiting the continuity of the embedding $\mathcal{V} \subset \mathcal{H}$ and the facts $v_{\tau} \rightarrow u^{\prime}$ weakly in $\mathcal{V}$, and $v_{\tau}^{\prime}(t)=\bar{z}_{\tau}(t)$ for all $t \in(0, T)$, and applying [48, Proposition 23.19], we conclude that $w=v^{\prime}=u^{\prime \prime}$. Therefore, (3.41) is valid.

We turn to show (3.42) and (3.43). From $H(F)$ and Lemma 6, we have

$$
\beta_{\tau}(t)=\beta_{0}+\int_{0}^{t} F\left(s, M \bar{u}_{\tau}(s), \beta_{\tau}(s)\right) d s,
$$

for all $t \in[0, T]$. Analogously, for another time step $h=T / N_{2}$ with $N_{2} \in \mathbb{N}$, we can write

$$
\beta_{h}(t)=\beta_{0}+\int_{0}^{t} F\left(s, M \bar{u}_{h}(s), \beta_{h}(s)\right) d s
$$


for all $t \in[0, T]$. Subtracting the last two equations and bounding, we get

$$
\begin{aligned}
\left\|\beta_{\tau}(t)-\beta_{h}(t)\right\|_{Y} & \leq \int_{0}^{t}\left\|F\left(t, M \bar{u}_{\tau}(s), \beta_{\tau}(s)\right)-F\left(t, M \bar{u}_{h}(s), \beta_{h}(s)\right)\right\|_{Y} d s \\
& \leq L_{F} \int_{0}^{t}\left\|M\left(\bar{u}_{\tau}(s)-\bar{u}_{h}(s)\right)\right\|_{X} d s+L_{F} \int_{0}^{t}\left\|\beta_{\tau}(s)-\beta_{h}(s)\right\|_{Y} d s,
\end{aligned}
$$

for all $t \in[0, T]$. Apply a Gronwall's lemma, see [44, Lemma 2.31], and the Hölder's inequality, to get

$$
\begin{aligned}
\left\|\beta_{\tau}(t)-\beta_{h}(t)\right\|_{Y} & \leq L_{F}\left(1+T L_{F} e^{L_{F} T}\right) \int_{0}^{t}\left\|M\left(\bar{u}_{\tau}(s)-\bar{u}_{h}(s)\right)\right\|_{X} d s \\
& \leq L_{F}\left(1+T L_{F} e^{L_{F} T}\right) \int_{0}^{T}\left\|M\left(\bar{u}_{\tau}(s)-\bar{u}_{h}(s)\right)\right\|_{X} d s \\
& \leq L_{F}\left(1+T L_{F} e^{L_{F} T}\right) \sqrt{T}\left\|\mathcal{M}\left(\bar{u}_{\tau}-\bar{u}_{h}\right)\right\|_{\mathcal{X}}
\end{aligned}
$$

for all $t \in[0, T]$. Further, directly from (3.2), we deduce

$$
\left\|\beta_{\tau}^{\prime}-\beta_{h}^{\prime}\right\|_{\mathcal{Y}}^{2} \leq C\left\|\mathcal{M}\left(\bar{u}_{\tau}-\bar{u}_{h}\right)\right\|_{\mathcal{X}}^{2}+C\left\|\beta_{\tau}-\beta_{h}\right\|_{\mathcal{Y}}^{2} .
$$

From the last two inequalities, we infer

$$
\left\|\beta_{\tau}-\beta_{h}\right\|_{W^{1,2}(0, T ; Y)} \leq C\left\|\mathcal{M}\left(\bar{u}_{\tau}-\bar{u}_{h}\right)\right\|_{\mathcal{X}}
$$

Hence, using the fact that $\mathcal{M}: M^{2,2}\left(0, T ; V, V^{*}\right) \subset \mathcal{V} \rightarrow \mathcal{X}$ is compact by $H(M)$, and $\left\{\bar{u}_{\tau}\right\}$ is bounded in $M^{2,2}\left(0, T ; V, V^{*}\right)$ (see (3.32)) with $\bar{u}_{\tau} \rightarrow u$ weakly in $\mathcal{V}$, we see that $\left\{\beta_{\tau}\right\}$ is a Cauchy sequence in $W^{1,2}(0, T ; Y)$. The completeness of $W^{1,2}(0, T ; Y)$ implies that there exists a function $\beta \in W^{1,2}(0, T ; Y)$ such that $\beta_{\tau} \rightarrow \beta$ in $W^{1,2}(0, T ; Y)$. Thus, (3.42) holds. Moreover, (3.43) follows from (3.33), (3.37) and Proposition 2.

It remains to show that $(u, \beta) \in \mathcal{V} \times W^{1,2}(0, T ; Y)$ is a solution to Problem 4. Since the operator $\mathcal{M}: M^{2,2}\left(0, T ; V, V^{*}\right) \subset \mathcal{V} \rightarrow \mathcal{X}$ is compact, $f_{\tau} \rightarrow f$ in $\mathcal{V}^{*}$ as $\tau \rightarrow 0$, see [7, Lemma 3.3], we use convergences (3.37), (3.40), (3.41) and (3.43) to deduce that

$$
\left\{\begin{array}{l}
\left(\bar{z}_{\tau}, w-\bar{v}_{\tau}\right)_{\mathcal{H}} \rightarrow\left(u^{\prime \prime}, w-u^{\prime}\right)_{\mathcal{H}}, \\
\left\langle\xi_{\tau}, \mathcal{M}\left(w-\bar{v}_{\tau}\right)\right\rangle_{\mathcal{X}^{*} \times \mathcal{X}} \rightarrow\left\langle\xi, \mathcal{M}\left(w-u^{\prime}\right)\right\rangle_{\mathcal{X}^{*} \times \mathcal{X}}, \\
\left\langle f_{\tau}, w-\bar{v}_{\tau}\right\rangle_{\mathcal{V}^{*} \times \mathcal{V}} \rightarrow\left\langle f, w-u^{\prime}\right\rangle_{\mathcal{V}^{*} \times \mathcal{V},}
\end{array}\right.
$$

for all $w \in \mathcal{V}$. From (3.35), (3.37), the continuity of $B$ and the fact $\left\|u_{\tau}-\bar{u}_{\tau}\right\|_{\mathcal{V}} \rightarrow 0$ as $\tau \rightarrow 0$,

$$
\left\langle\mathcal{B} \bar{u}_{\tau}, w\right\rangle_{\mathcal{V}^{*} \times \mathcal{V}} \rightarrow\langle\mathcal{B} u, w\rangle_{\mathcal{V}^{*} \times \mathcal{V}} \text { and }\left\langle\mathcal{B}\left(u_{\tau}-\bar{u}_{\tau}\right), \bar{v}_{\tau}\right\rangle_{\mathcal{V}^{*} \times \mathcal{V}} \rightarrow 0
$$

where $\mathcal{B}: \mathcal{V} \rightarrow \mathcal{V}^{*}$ is the Nemytskii operator corresponding to $B$ defined by $\mathcal{B}(u)(t)=B u(t)$ for $t \in[0, T]$ and $u \in \mathcal{V}$. The weak convergence $u_{\tau} \rightarrow u$ and $u_{\tau}^{\prime}=\bar{v}_{\tau} \rightarrow u^{\prime}$ in $\mathcal{V}$ implies that $u_{\tau} \rightarrow u$ weakly in $W^{1,2}(0, T ; V)$. The latter combined with the continuity of the embedding $W^{1,2}(0, T ; V) \subset C(0, T ; V)$ and [34, Lemma $\left.4(\mathrm{a})\right]$ entails $u_{\tau}(t) \rightarrow u(t)$ weakly in $V$ for all $t \in$ $[0, T]$. In particular, we have $u_{\tau}(T) \rightarrow u(T)$ weakly in $V$. Since the function $V \ni u \mapsto\langle B u, u\rangle \in \mathbb{R}$ is weakly l.s.c. (being convex and continuous), one has

$$
\langle B u(T), u(T)\rangle \leq \liminf _{\tau \rightarrow 0}\left\langle B u_{\tau}(T), u_{\tau}(T)\right\rangle .
$$


Combining the above inequality with (3.45), we obtain

$$
\begin{aligned}
\limsup _{\tau \rightarrow 0}\left\langle\mathcal{B} \bar{u}_{\tau}, w-\bar{v}_{\tau}\right\rangle_{\mathcal{V}^{*} \times \mathcal{V}}= & \limsup _{\tau \rightarrow 0}\left[\left\langle\mathcal{B} \bar{u}_{\tau}, w\right\rangle_{\mathcal{V}^{*} \times \mathcal{V}}-\frac{1}{2}\left(\left\langle B u_{\tau}(T), u_{\tau}(T)\right\rangle-\langle B u(0), u(0)\rangle\right)\right. \\
& \left.+\left\langle\mathcal{B}\left(u_{\tau}-\bar{u}_{\tau}\right), \bar{v}_{\tau}\right\rangle_{\mathcal{V}^{*} \times \mathcal{V}}\right] \\
& \leq \limsup _{\tau \rightarrow 0}\left\langle\mathcal{B} \bar{u}_{\tau}, w\right\rangle_{\mathcal{V}^{*} \times \mathcal{V}}+\limsup _{\tau \rightarrow 0}\left\langle\mathcal{B}\left(u_{\tau}-\bar{u}_{\tau}\right), \bar{v}_{\tau}\right\rangle_{\mathcal{V}^{*} \times \mathcal{V}} \\
& -\frac{1}{2} \liminf _{\tau \rightarrow 0}\left\langle B u_{\tau}(T), u_{\tau}(T)\right\rangle+\frac{1}{2}\langle B u(0), u(0)\rangle \\
& \leq\langle\mathcal{B} u, w\rangle_{\mathcal{V}^{*} \times \mathcal{V}}-\frac{1}{2}(\langle B u(T), u(T)\rangle-\langle B u(0), u(0)\rangle) \\
= & \langle\mathcal{B} u, w\rangle_{\mathcal{V}^{*} \times \mathcal{V}}-\int_{0}^{T}\left\langle B u(t), u^{\prime}(t)\right\rangle d t=\left\langle\mathcal{B} u, w-u^{\prime}\right\rangle_{\mathcal{V}^{*} \times \mathcal{V} .}
\end{aligned}
$$

Next, we will show that the function $\Phi: \mathcal{V} \rightarrow \mathbb{R} \cup\{+\infty\}$ defined by

$$
\Phi(v)=\int_{0}^{T} \varphi(v(t)) d t \quad \text { for all } v \in \mathcal{V}
$$

is proper, convex and 1.s.c. In fact, hypothesis $H(0)(\mathrm{i})$ implies $v_{0} \in \operatorname{dom} \Phi$, that is, $\Phi\left(v_{0}\right)<+\infty$. The convexity of $\Phi$ is a consequence of the convexity for $\varphi$. Let $\left\{w_{n}\right\} \subset \mathcal{V}, w \in \mathcal{V}$ and $w_{n} \rightarrow w$ in $\mathcal{V}$. Again, we use the fact that $\varphi$ is bounded below by an affine functional: there exist $k_{1}, k_{2} \geq 0$ such that

$$
\varphi(v) \geq-k_{1}\|v\|-k_{2} \quad \text { for all } v \in V
$$

Hence, we have

$$
\int_{0}^{T} \varphi\left(w_{n}(t)\right) d t \geq-k_{1} \int_{0}^{T}\left\|w_{n}(t)\right\| d t-k_{2} T \geq-k_{2} T-k_{1} \sqrt{T}\left\|w_{n}\right\|_{\mathcal{V}} \geq C .
$$

Applying the converse Lebesgue's dominated convergence theorem ([35, Theorem 2.39]), by passing to a subsequence if necessary, we may suppose that $w_{n}(t) \rightarrow w(t)$ in $V$ for a.e. $t \in(0, T)$. Now, the lower semicontinuity of $\varphi$ and Fatou's lemma, [35, Theorem 1.64], entail

$$
\Phi(w)=\int_{0}^{T} \varphi(w(t)) d t \leq \int_{0}^{T} \liminf _{n \rightarrow \infty} \varphi\left(w_{n}(t)\right) d t \leq \liminf _{n \rightarrow \infty} \int_{0}^{T} \varphi\left(w_{n}(t)\right) d t=\liminf _{n \rightarrow \infty} \Phi\left(w_{n}\right) .
$$

Thus $\Phi$ is 1.s.c. Because it is convex, it is also weakly sequentially lower semicontinuous on $\mathcal{V}$. Hence, we have $\Phi\left(u^{\prime}\right) \leq \liminf _{\tau \rightarrow 0} \Phi\left(\bar{v}_{\tau}\right)$.

Now, we show that for all $w \in \mathcal{V}$

$$
\limsup _{\tau \rightarrow 0}\left\langle\mathcal{A} \bar{v}_{\tau}, w-\bar{v}_{\tau}\right\rangle_{\mathcal{V}^{*} \times \mathcal{V}} \leq\left\langle\mathcal{A} u^{\prime}, w-u^{\prime}\right\rangle_{\mathcal{V}^{*} \times \mathcal{V}}
$$

or equivalently

$$
\liminf _{\tau \rightarrow 0}\left\langle\mathcal{A} \bar{v}_{\tau}, \bar{v}_{\tau}-w\right\rangle_{\mathcal{V}^{*} \times \mathcal{V}} \geq\left\langle\mathcal{A} u^{\prime}, u^{\prime}-w\right\rangle_{\mathcal{V}^{*} \times \mathcal{V}}
$$


where the operator $\mathcal{A}: \mathcal{V} \rightarrow \mathcal{V}^{*}$ stands for the Nemytskii operator corresponding to $A$ defined by

$$
\mathcal{A}(u)(t)=A u(t) \text { for a.e. } t \in[0, T] \text { and } u \in \mathcal{V} .
$$

To prove inequality (3.48), first, we shall show that

$$
\limsup _{\tau \rightarrow 0}\left\langle\mathcal{A} \bar{v}_{\tau}, \bar{v}_{\tau}-u^{\prime}\right\rangle_{\mathcal{V}^{*} \times \mathcal{V}} \leq 0 .
$$

We rewrite the inequality (3.5) as

$$
\begin{gathered}
\left(\bar{z}_{\tau}(t), v-\bar{v}_{\tau}(t)\right)_{H}+\left\langle A \bar{v}_{\tau}(t)+B \bar{u}_{\tau}(t), v-\bar{v}_{\tau}(t)\right\rangle+\left\langle\xi_{\tau}(t), M\left(v-\bar{v}_{\tau}(t)\right)\right\rangle_{X^{*} \times X} \\
\quad+\varphi(v)-\varphi\left(\bar{v}_{\tau}(t)\right) \geq\left\langle f_{\tau}(t), v-\bar{v}_{\tau}(t)\right\rangle,
\end{gathered}
$$

for all $v \in V$ and a.e. $t \in(0, T)$. Choosing $v=u^{\prime}(t)$ in the above inequality and then integrating it on $(0, T)$, we obtain

$$
\begin{aligned}
\left\langle\mathcal{A} \bar{v}_{\tau}, \bar{v}_{\tau}-u^{\prime}\right\rangle_{\mathcal{V}^{*} \times \mathcal{V} \leq} & \left(\bar{z}_{\tau}, u^{\prime}-\bar{v}_{\tau}\right)_{\mathcal{H}}+\left\langle\mathcal{B} \bar{u}_{\tau}, u^{\prime}-\bar{v}_{\tau}\right\rangle_{\mathcal{V}^{*} \times \mathcal{V}}+\left\langle\xi_{\tau}, \mathcal{M}\left(u^{\prime}-\bar{v}_{\tau}\right)\right\rangle_{\mathcal{X}^{*} \times \mathcal{X}} \\
& +\Phi\left(u^{\prime}\right)-\Phi\left(\bar{v}_{\tau}\right)+\left\langle f_{\tau}, \bar{v}_{\tau}-u^{\prime}\right\rangle_{\mathcal{V}^{*} \times \mathcal{V}}
\end{aligned}
$$

Then, we take into account (3.44)-(3.46) and pass to the upper limit, as $\tau \rightarrow 0$,

$$
\begin{aligned}
& \limsup _{\tau \rightarrow 0}\left\langle\mathcal{A} \bar{v}_{\tau}, \bar{v}_{\tau}-u^{\prime}\right\rangle_{\mathcal{V}^{*} \times \mathcal{V}} \\
& \leq \limsup _{\tau \rightarrow 0}\left(\bar{z}_{\tau}, u^{\prime}-\bar{v}_{\tau}\right)_{\mathcal{H}}+\limsup _{\tau \rightarrow 0}\left\langle\mathcal{B} \bar{u}_{\tau}, u^{\prime}-\bar{v}_{\tau}\right\rangle_{\mathcal{V}^{*} \times \mathcal{V}}+\Phi\left(u^{\prime}\right)-\liminf _{\tau \rightarrow 0} \Phi\left(\bar{v}_{\tau}\right) \\
& \quad+\limsup _{\tau \rightarrow 0}\left\langle\xi_{\tau}, \mathcal{M}\left(u^{\prime}-\bar{v}_{\tau}\right)\right\rangle_{\mathcal{X}^{*} \times \mathcal{X}}+\limsup _{\tau \rightarrow 0}\left\langle f_{\tau}, \bar{v}_{\tau}-u^{\prime}\right\rangle_{\mathcal{V}^{*} \times \mathcal{V}} \\
& \quad \leq 0,
\end{aligned}
$$

which implies (3.49). Invoking [25, Lemma 1], $H(A),(3.37),(3.49)$ and (3.33), we obtain $\mathcal{A} \bar{v}_{\tau} \rightarrow$ $\mathcal{A} u^{\prime}$ weakly in $\mathcal{V}^{*}$ and (3.48) holds.

Next, combining (3.44), (3.46) and (3.47), for all $w \in \mathcal{V}$, we have

$$
\begin{aligned}
0 \leq & \limsup _{\tau \rightarrow 0}\left(\left(\bar{z}_{\tau}, w-\bar{v}_{\tau}\right)_{\mathcal{H}}+\left\langle\mathcal{A} \bar{v}_{\tau}, w-\bar{v}_{\tau}\right\rangle_{\mathcal{V}^{*} \times \mathcal{V}}+\left\langle\mathcal{B} \bar{u}_{\tau}, w-\bar{v}_{\tau}\right\rangle_{\mathcal{V}^{*} \times \mathcal{V}}\right. \\
& \left.+\left\langle\xi_{\tau}, \mathcal{M}\left(w-\bar{v}_{\tau}\right)\right\rangle_{\mathcal{X}^{*} \times \mathcal{X}}+\Phi(w)-\Phi\left(\bar{v}_{\tau}\right)-\left\langle f_{\tau},\left(w-\bar{v}_{\tau}\right)\right\rangle_{\mathcal{V}^{*} \times \mathcal{V}}\right) \\
\leq & \left(u^{\prime \prime}, w-u^{\prime}\right)_{\mathcal{H}}+\left\langle\mathcal{A} u^{\prime}, w-u^{\prime}\right\rangle_{\mathcal{V}^{*} \times \mathcal{V}}+\left\langle\mathcal{B} u, w-u^{\prime}\right\rangle_{\mathcal{V}^{*} \times \mathcal{V}} \\
& +\left\langle\xi, \mathcal{M}\left(w-u^{\prime}\right)\right\rangle_{\mathcal{X}^{*} \times \mathcal{X}}+\Phi(w)-\Phi\left(u^{\prime}\right)-\left\langle f, w-u^{\prime}\right\rangle_{\mathcal{V}^{*} \times \mathcal{V}}
\end{aligned}
$$

Finally, we will demonstrate that $\xi(t) \in \partial J\left(\beta(t), M u^{\prime}(t)\right)$ for a.e. $t \in(0, T)$. Since $\mathcal{M} \bar{v}_{\tau} \rightarrow \mathcal{M} u^{\prime}$ in $\mathcal{X}$, from the converse Lebesgue's dominated convergence theorem, [35, Theorem 1.64], by passing to a subsequence if necessary, we may assume that $M \bar{v}_{\tau}(t) \rightarrow M u^{\prime}(t)$ in $X$ for a.e. $t \in(0, T)$. Since $\beta_{\tau} \rightarrow \beta$ in $W^{1,2}(0, T ; Y), \beta_{\tau}(t) \rightarrow \beta(t)$ in $Y$ for all $t \in[0, T]$. Now $\xi_{\tau}(t) \in$ $\partial J\left(\beta_{\tau}(t), M \bar{v}_{\tau}(t)\right)$ for a.e. $t \in(0, T), \xi_{\tau} \rightarrow \xi$ weakly in $\mathcal{X}^{*}$, and $\partial J$ is upper semicontinuous by Lemma 5. We can apply [2, Theorem 1, p. 60] to get

$$
\xi(t) \in \partial J\left(\beta(t), M u^{\prime}(t)\right) \text { for a.e. } t \in(0, T) .
$$

Applying Proposition 8 , we conclude that $(u, \beta) \in \mathcal{V} \times \mathcal{Y}$ is a solution to Problem 4. 
We complete this section with results on uniqueness and regularity of the solution to Problem 4.

Theorem 14 Assume $H(A), H(B), H(M), H(J), H(\varphi), H(F), H(f)$ and $H(0)$. Then, Problem 4 has a unique solution.

Proof Assume that $\left(u_{1}, \beta_{1}\right),\left(u_{2}, \beta_{2}\right) \in \mathcal{V} \times W^{1,2}(0, T ; Y)$ are two solutions to Problem 4. We have

$$
\left\{\begin{array}{l}
\left\langle u_{1}^{\prime \prime}(t), v-u_{1}^{\prime}(t)\right\rangle+\left\langle A u_{1}^{\prime}(t)+B u_{1}(t), v-u_{1}^{\prime}(t)\right\rangle+\varphi(v)-\varphi\left(u_{1}^{\prime}(t)\right) \\
\quad+\left\langle\xi_{1}(t), M\left(v-u_{1}^{\prime}(t)\right)\right\rangle_{X^{*} \times X} \geq\left\langle f(t), v-u_{1}^{\prime}(t)\right\rangle \text { for all } v \in V, \text { a.e. } t \in(0, T), \\
\xi_{1}(t) \in \partial J\left(\beta_{1}(t), M u_{1}^{\prime}(t)\right) \text { for a.e. } t \in(0, T), \\
\beta_{1}^{\prime}(t)=F\left(t, M u_{1}(t), \beta_{1}(t)\right) \text { for a.e. } t \in(0, T), \\
u_{1}(0)=u_{0}, \quad u_{1}^{\prime}(0)=v_{0}, \quad \beta_{1}(0)=\beta_{0}
\end{array}\right.
$$

and

$$
\left\{\begin{array}{l}
\left\langle u_{2}^{\prime \prime}(t), v-u_{2}^{\prime}(t)\right\rangle+\left\langle A u_{2}^{\prime}(t)+B u_{2}(t), v-u_{2}^{\prime}(t)\right\rangle+\varphi(v)-\varphi\left(u_{2}^{\prime}(t)\right) \\
\quad+\left\langle\xi_{2}(t), M\left(v-u_{2}^{\prime}(t)\right)\right\rangle_{X^{*} \times X} \geq\left\langle f(t), v-u_{2}^{\prime}(t)\right\rangle \quad \text { for all } v \in V, \text { a.e. } t \in(0, T), \\
\xi_{2}(t) \in \partial J\left(\beta_{2}(t), M u_{2}^{\prime}(t)\right) \quad \text { for a.e. } t \in(0, T), \\
\beta_{2}^{\prime}(t)=F\left(t, M u_{2}(t), \beta_{2}(t)\right) \quad \text { for a.e. } t \in(0, T), \\
u_{2}(0)=u_{0}, \quad u_{2}^{\prime}(0)=v_{0}, \quad \beta_{2}(0)=\beta_{0} .
\end{array}\right.
$$

Taking $v=u_{2}^{\prime}(t)$ in (3.50) and $v=u_{1}^{\prime}(t)$ in (3.51), we add the resulting inequalities to get

$$
\begin{aligned}
& \left\langle u_{1}^{\prime \prime}(t)-u_{2}^{\prime \prime}(t), u_{1}^{\prime}(t)-u_{2}^{\prime}(t)\right\rangle+\left\langle A u_{1}^{\prime}(t)-A u_{2}^{\prime}(t), u_{1}^{\prime}(t)-u_{2}^{\prime}(t)\right\rangle \\
& \quad+\left\langle B u_{1}(t)-B u_{2}(t), u_{1}^{\prime}(t)-u_{2}^{\prime}(t)\right\rangle+\left\langle\xi_{1}(t)-\xi_{2}(t), M\left(u_{1}^{\prime}(t)-u_{2}^{\prime}(t)\right)\right\rangle_{X^{*} \times X} \leq 0 .
\end{aligned}
$$

Next, we integrate the above inequality on $(0, t)$, for any $t \in(0, T)$, and obtain

$$
\begin{aligned}
& \frac{1}{2}\left\|u_{1}^{\prime}(t)-u_{2}^{\prime}(t)\right\|_{H}^{2}+m_{A} \int_{0}^{t}\left\|u_{1}^{\prime}(s)-u_{2}^{\prime}(s)\right\|^{2} d s-c_{A} \int_{0}^{t}\left\|u_{1}^{\prime}(s)-u_{2}^{\prime}(s)\right\|_{H}^{2} d s \\
& +\frac{\varrho_{B}}{2}\left\|u_{1}(t)-u_{2}(t)\right\|^{2}-m_{J}\|M\|_{\mathcal{L}(V, X)}^{2} \int_{0}^{t}\left\|u_{1}^{\prime}(s)-u_{2}^{\prime}(s)\right\|^{2} d s \\
& \quad-m_{J} \int_{0}^{t}\left\|M\left(u_{1}^{\prime}(s)-u_{2}^{\prime}(s)\right)\right\|_{X}\left\|\beta_{1}(s)-\beta_{2}(s)\right\|_{Y} d s \leq 0 .
\end{aligned}
$$

From the Cauchy's inequality and inequality (3.3), we have

$$
\begin{aligned}
& m_{J} \int_{0}^{t}\left\|M\left(u_{1}^{\prime}(s)-u_{2}^{\prime}(s)\right)\right\|_{X}\left\|\beta_{1}(s)-\beta_{2}(s)\right\|_{Y} d s \\
& \quad \leq m_{J} c_{F} \int_{0}^{t} \int_{0}^{s}\left\|u_{1}^{\prime}(s)-u_{2}^{\prime}(s)\right\|\left\|u_{1}(\zeta)-u_{2}(\zeta)\right\| d \zeta d s
\end{aligned}
$$




$$
\begin{aligned}
& \leq \frac{m_{J}^{2} c_{F}^{2}}{4 \varepsilon} \int_{0}^{t} \int_{0}^{s}\left\|u_{1}(\zeta)-u_{2}(\zeta)\right\|^{2} d \zeta d s+\varepsilon T \int_{0}^{t}\left\|u_{1}^{\prime}(s)-u_{2}^{\prime}(s)\right\|^{2} d s \\
& \leq \frac{m_{J}^{2} c_{F}^{2} T}{4 \varepsilon} \int_{0}^{t}\left\|u_{1}(s)-u_{2}(s)\right\|^{2} d s+\varepsilon T \int_{0}^{t}\left\|u_{1}^{\prime}(s)-u_{2}^{\prime}(s)\right\|^{2} d s,
\end{aligned}
$$

where $\varepsilon=\frac{1}{2 T}\left(m_{A}-m_{J}\|M\|_{\mathcal{L}(V, X)}^{2}\right)$. Using the last inequality in (3.52), we deduce that

$$
\begin{gathered}
\frac{1}{2}\left\|u_{1}^{\prime}(t)-u_{2}^{\prime}(t)\right\|_{H}^{2}+\left(m_{A}-m_{J}\|M\|_{\mathcal{L}(V, X)}^{2}-\varepsilon T\right) \int_{0}^{t}\left\|u_{1}^{\prime}(s)-u_{2}^{\prime}(s)\right\|^{2} d s \\
+\frac{\varrho_{B}}{2}\left\|u_{1}(t)-u_{2}(t)\right\|^{2} \leq C_{5} \int_{0}^{t}\left\|u_{1}^{\prime}(s)-u_{2}^{\prime}(s)\right\|_{H}^{2}+\left\|u_{1}(s)-u_{2}(s)\right\|^{2} d s
\end{gathered}
$$

for all $t \in(0, T)$, where $C_{5}$ is given by

$$
C_{5}=\max \left\{c_{A}, \frac{m_{J}^{2} c_{F}^{2} T}{4 \varepsilon}\right\} .
$$

Finally, we apply the Gronwall's lemma, see [44, Lemma 2.31], to derive $u_{1}=u_{2}$. Moreover, Lemma 6 guarantees that $\beta_{1}=\beta_{2}$, which completes the proof of the theorem.

Theorem 15 Assume $H(A), H(B), H(J), H(M), H(F), H(\varphi), H(f)$ and $H(0)$. If $u$ is a solution to Problem 4, then $u:[0, T] \rightarrow V$ is Hölder continuous with exponent $\frac{1}{2}$.

Proof By Theorem 13, it is known that $u, u^{\prime} \in \mathcal{V}$. Applying the Hölder's inequality, we have

$$
\left\|u\left(t_{1}\right)-u\left(t_{2}\right)\right\| \leq \int_{t_{1}}^{t_{2}}\left\|u^{\prime}(s)\right\| d s \leq\left|t_{1}-t_{2}\right|^{\frac{1}{2}}\left(\int_{0}^{T}\left\|u^{\prime}(t)\right\|^{2} d t\right)^{\frac{1}{2}},
$$

for all $t_{1}, t_{2} \in[0, T]$, which implies the desired regularity.

\section{A dynamic viscoelastic frictional contact problem}

In this section, we illustrate the applicability of theoretical results from Section 3 in the study of a new dynamic frictional contact model for a viscoelastic material with adhesion effects. The contact boundary conditions in this model are described by a generalised normal damped response condition with a unilateral constraint together with a multivalued friction law.

The physical setting of the dynamic contact process is described as follows. Assume that the viscoelastic body occupies a bounded and open domain $\Omega$ in $\mathbb{R}^{d}(d=2,3)$ with a Lipschitz continuous boundary $\Gamma:=\partial \Omega$. The boundary $\partial \Omega$ is given by $\Gamma=\Gamma_{D} \cup \Gamma_{N} \cup \Gamma_{C}$ such that $\Gamma_{D}$, $\Gamma_{N}$ and $\Gamma_{C}$ are pairwise disjoint and measurable with meas $\left(\Gamma_{D}\right)>0$. We are interested in the evolution of the body in a finite time interval $(0, T)$.

We adopt the following standard notation. The unit outward normal vector on boundary and the position vector in the body are denoted by $\boldsymbol{v}=\left(v_{i}\right)$ and $\boldsymbol{x} \in \bar{\Omega}=\Omega \cup \partial \Omega$, respectively. The indices $i, j, k, l$ run from 1 to $d$, and unless stated otherwise, the summation convention over repeated indices is used. For simplicity, we often will not indicate explicitly the dependence of the variables on $\boldsymbol{x}$. Let $\left(\mathbb{S}^{d},\|\cdot\|_{\mathbb{S}^{d}}\right)$ be the space of second-order symmetric tensors on $\mathbb{R}^{d}$. Throughout this section, the inner products and norms in $\mathbb{R}^{d}$ and $\mathbb{S}^{d}$ are denoted by $\boldsymbol{u} \cdot \boldsymbol{v}=u_{i} v_{i}$, 
$\|\boldsymbol{v}\|_{\mathbb{R}^{d}}=(\boldsymbol{v} \cdot \boldsymbol{v})^{\frac{1}{2}}$ for $\boldsymbol{u}=\left(u_{i}\right), \boldsymbol{v}=\left(v_{i}\right) \in \mathbb{R}^{d}$ and $\boldsymbol{\sigma}: \boldsymbol{\tau}=\sigma_{i j} \tau_{i j},\|\boldsymbol{\tau}\|_{\mathbb{S}^{d}}=(\boldsymbol{\tau}: \boldsymbol{\tau})^{\frac{1}{2}}$ for $\boldsymbol{\sigma}=\left(\sigma_{i j}\right), \boldsymbol{\tau}=$ $\left(\tau_{i j}\right) \in \mathbb{S}^{d}$, respectively. In addition, we use the notation $\boldsymbol{u}=\left(u_{i}\right), \boldsymbol{\sigma}=\left(\sigma_{i j}\right)$, and

$$
\boldsymbol{\varepsilon}(\boldsymbol{u})=\left(\varepsilon_{i j}(\boldsymbol{u})\right), \quad \varepsilon_{i j}(\boldsymbol{u})=\frac{1}{2}\left(u_{i, j}+u_{j, i}\right), \quad i, j=1, \ldots, d,
$$

to denote the displacement vector, the stress tensor and the linearised strain tensor, respectively. For a vector $\boldsymbol{w}$ defined on the boundary, its normal and tangential components are $w_{v}=\boldsymbol{w} \cdot \boldsymbol{v}$ and $\boldsymbol{w}_{\tau}=\boldsymbol{w}-w_{\nu} \boldsymbol{v}$. For the stress tensor $\boldsymbol{\sigma}$, its normal and tangential components on the boundary are defined by $\sigma_{v}=(\boldsymbol{\sigma} \boldsymbol{v}) \cdot \boldsymbol{v}$ and $\boldsymbol{\sigma}_{\tau}=\boldsymbol{\sigma} \boldsymbol{v}-\sigma_{v} \boldsymbol{v}$, respectively. Let $\mathcal{Q}=\Omega \times(0, T), \Sigma_{D}=\Gamma_{D} \times$ $(0, T), \Sigma_{N}=\Gamma_{N} \times(0, T)$ and $\Sigma_{C}=\Gamma_{C} \times(0, T)$.

We are now in a position to present the classical formulation of the contact problem.

Problem 16 Find a displacement field $\boldsymbol{u}: \mathcal{Q} \rightarrow \mathbb{R}^{d}$, a stress field $\boldsymbol{\sigma}: \mathcal{Q} \rightarrow \mathbb{S}^{d}$ and a bonding field $\beta: \Gamma_{C} \times(0, T) \rightarrow[0,1]$ such that

$$
\begin{aligned}
& \boldsymbol{\sigma}(t)=\mathcal{C}\left(\boldsymbol{\varepsilon}\left(\boldsymbol{u}^{\prime}(t)\right)\right)+\mathcal{E}(\boldsymbol{\varepsilon}(\boldsymbol{u}(t))) \quad \text { in } \mathcal{Q}, \\
& \boldsymbol{u}^{\prime \prime}(t)-\operatorname{Div} \boldsymbol{\sigma}(t)=\boldsymbol{f}_{0}(t) \quad \text { in } \mathcal{Q} \text {, } \\
& \boldsymbol{u}(t)=\mathbf{0}_{\mathbb{R}^{d}} \quad \text { on } \Sigma_{D} \text {, } \\
& \boldsymbol{\sigma}(t) \boldsymbol{v}=\boldsymbol{f}_{N}(t) \quad \text { on } \Sigma_{N} \text {, } \\
& \left\{\begin{array}{l}
u_{\nu}^{\prime}(t) \leq g, \sigma_{\nu}(t)+\xi(t) \leq 0 \\
\left(u_{\nu}^{\prime}(t)-g\right)\left(\sigma_{\nu}(t)+\xi(t)\right)=0 \\
\xi(t) \in \partial j_{v}\left(\beta(t), u_{\nu}^{\prime}(t)\right)
\end{array} \quad \text { on } \Sigma_{C},\right. \\
& -\sigma_{\tau}(t) \in \partial j_{\tau}\left(\beta(t), \boldsymbol{u}_{\tau}^{\prime}(t)\right) \quad \text { on } \Sigma_{C} \text {, } \\
& \beta^{\prime}(t)=F(t, \boldsymbol{u}(t), \beta(t)) \quad \text { on } \Sigma_{C} \text {, } \\
& \beta(0)=\beta_{0} \quad \text { on } \Gamma_{C} \text {, } \\
& \boldsymbol{u}(0)=\boldsymbol{u}_{0}, \quad \boldsymbol{u}^{\prime}(0)=\boldsymbol{v}_{0} \quad \text { in } \Omega .
\end{aligned}
$$

Let us briefly comment on the equations and conditions in Problem 16. The equation (4.1) is the well-known Kelvin-Voigt viscoelastic constitutive law in which $\mathcal{C}$ and $\mathcal{E}$ stand for a nonlinear viscosity operator and a linear elasticity operator, respectively. The equation of motion (4.2) reflects the fact that the contact process is dynamic. Here, 'Div' is the divergence operator given by

$$
\operatorname{Div} \boldsymbol{\sigma}=\left(\sigma_{i j, j}\right)=\left(\frac{\partial \sigma_{i j}}{\partial x_{j}}\right)
$$

and $\boldsymbol{f}_{0}$ is the density of applied volume forces (e.g., gravity). The boundary conditions (4.3) and (4.4) mean that the body is clamped on $\Gamma_{D}$, and it is subjected to the density $\boldsymbol{f}_{N}$ of surface tractions on $\Gamma_{N}$. The initial displacement and velocity are given in (4.9). The surface variable $\beta$ is usually called the bonding field or the adhesion field, see [14-16,21], which is a scale (dimensionless) function describing the pointwise fractional density of active bonds on the contact surface. In the model, we assume that the bonding field $\beta$ is governed by a non-linear evolution equation (4.7) on the surface of contact boundary $\Gamma_{C}$, and the function $F$ depends 
explicitly on the displacement field. The initial bonding field is assumed in (4.8). Moreover, we can distinguish three cases:

(i) $\beta=1$, when the adhesion is complete and all the bonds are active;

(ii) $\beta=0$, if all bonds are inactive and there is no adhesion effect;

(iii) $0<\beta<1$, when the adhesion is partial and a fracture $\beta$ of the bonds is active.

The contact condition (4.5) represents a multivalued normal damped response condition with unilateral constraint, which has been recently studied in [18]. It is worth mentioning that this boundary condition can be rewritten to the following equivalent form:

$$
-\sigma_{\nu}(t) \in \partial j_{v}\left(\beta(t), u_{v}^{\prime}(t)\right)+\partial_{c} I_{(-\infty, g]}\left(u_{v}^{\prime}(t)\right) \quad \text { on } \quad \Sigma_{C},
$$

where $I_{(-\infty, g]}: \mathbb{R} \rightarrow\{0,+\infty\}$ is the indicator function of the interval $(-\infty, g] \subset \mathbb{R}$,

$$
I_{(-\infty, g]}(r)=\left\{\begin{array}{rc}
0, & r \in(-\infty, g], \\
+\infty, & \text { otherwise, }
\end{array}\right.
$$

and $g>0$ is a positive constant. Note that (4.10) involves both the Clarke's generalised gradient and convex subdifferential, so this multivalued relation represents a more general boundary condition than the ones which are often found in contact models.

Example 17 Let $q: \mathbb{R} \times \mathbb{R} \rightarrow \mathbb{R}$ be a continuous function and $j: \mathbb{R} \times \mathbb{R} \rightarrow \mathbb{R}$ be defined by

$$
j(s, r)=\int_{0}^{r} q(s, t) d t \quad \text { for } s, r \in \mathbb{R} .
$$

It is clear that $j$ is a Lipschitz continuous function and $\partial j(s, r)=q(s, r)$ for all $s, r \in \mathbb{R}$. Moreover, if the potential $j_{v}=j$, then (4.5) reduces to ([44])

$$
u_{v}^{\prime}(t) \leq g, \quad \sigma_{v}(t)+q\left(\beta(t), u_{v}^{\prime}(t)\right) \leq 0, \quad\left(u_{v}^{\prime}(t)-g\right)\left(\sigma_{v}(t)+q\left(\beta(t), u_{v}^{\prime}\right)(t)\right)=0 \quad \text { on } \Sigma_{C} .
$$

In particular, when $q$ is independent of the bonding field $\beta$, that is, $q(s, r)=q(r)$ for all $s, r \in \mathbb{R}$, then (4.11) becomes ([13])

$$
u_{v}^{\prime}(t) \leq g, \quad \sigma_{v}(t)+q\left(u_{v}^{\prime}(t)\right) \leq 0, \quad\left(u_{v}^{\prime}(t)-g\right)\left(\sigma_{v}(t)+q\left(u_{v}^{\prime}(t)\right)\right)=0 \quad \text { on } \Sigma_{C} .
$$

Furthermore, with $q \equiv 0$ and $g \equiv 0$, the boundary condition (4.12) is reduced to the classical Signorini-type contact condition in velocity of the form, see [12, 22, 43, 45, 47]

$$
u_{v}^{\prime}(t) \leq 0, \quad \sigma_{v}(t) \leq 0, \quad \sigma_{v}(t) u_{v}^{\prime}(t)=0 \text { on } \Sigma_{C} .
$$

The general friction condition with adhesion (4.6) is described by the generalised gradient of a locally Lipschitz potential $j_{\tau}$, which is non-convex in general.

To derive a weak formulation of Problem 16, we introduce function spaces

$$
V=\left\{\boldsymbol{v} \in H^{1}\left(\Omega ; \mathbb{R}^{d}\right) \mid \boldsymbol{v}=\mathbf{0} \text { on } \Gamma_{D}\right\} \text { and } \mathcal{H}=L^{2}\left(\Omega ; \mathbb{S}^{d}\right) .
$$

Since meas $\left(\Gamma_{D}\right)>0, V$ is a Hilbert space endowed with the inner product

$$
(\boldsymbol{u}, \boldsymbol{v})_{V}=(\boldsymbol{\varepsilon}(\boldsymbol{u}), \boldsymbol{\varepsilon}(\boldsymbol{v}))_{\mathcal{H}}, \quad \boldsymbol{u}, \boldsymbol{v} \in V,
$$


and the associated norm $\|\cdot\|_{V} \cdot \mathcal{H}$ is a Hilbert space equipped with the inner product

$$
(\boldsymbol{\sigma}, \boldsymbol{\tau})_{\mathcal{H}}=\int_{\Omega} \sigma_{i j}(\boldsymbol{x}) \tau_{i j}(\boldsymbol{x}) d x, \quad \boldsymbol{\sigma}, \boldsymbol{\tau} \in \mathcal{H}
$$

and the norm $\|\cdot\|_{\mathcal{H}}$. The trace of an element $\boldsymbol{v} \in H^{1}\left(\Omega ; \mathbb{R}^{d}\right)$ will be denoted by the same symbol. The trace theorem states

$$
\|\boldsymbol{v}\|_{L^{2}\left(\Gamma_{C} ; \mathbb{R}^{d}\right)} \leq\|\gamma\|\|\boldsymbol{v}\|_{V} \quad \text { for all } \boldsymbol{v} \in V
$$

where $\gamma$ is the trace operator from $V$ to $L^{2}\left(\Gamma_{C} ; \mathbb{R}^{d}\right)$. The unilateral constraint condition (4.5) requires the use of the following set of admissible velocities

$$
K=\left\{\boldsymbol{v} \in V \mid v_{\nu} \leq g \text { on } \Gamma_{C}\right\}
$$

We now make assumptions on the problem data.

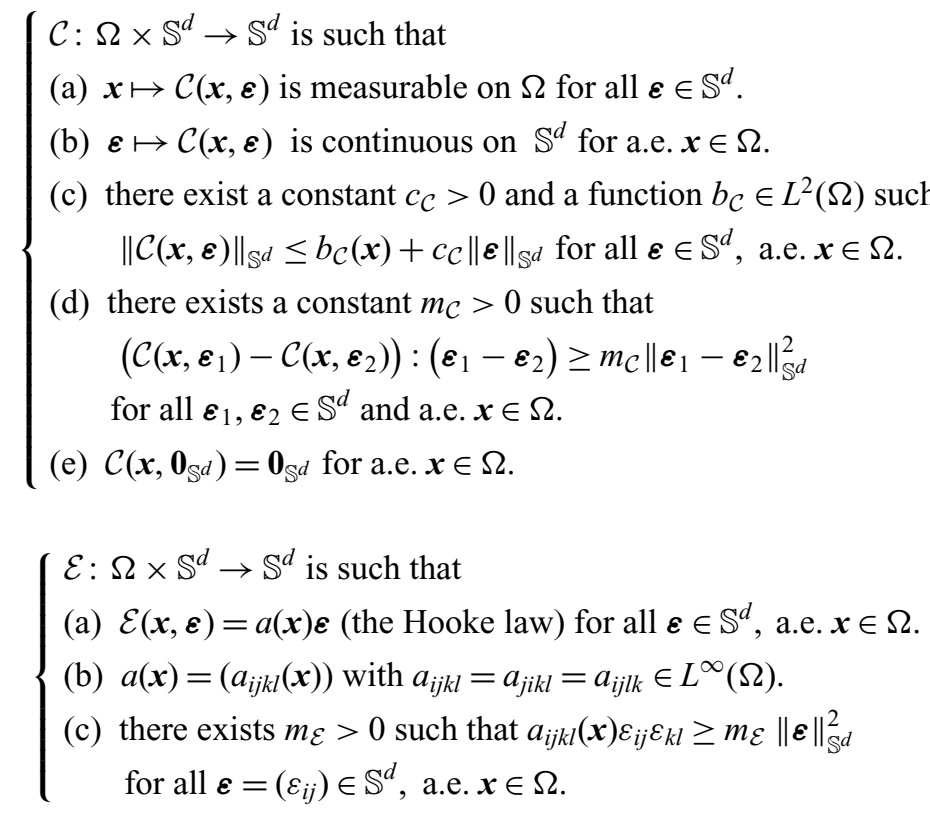

The normal and tangential potentials enjoy the following hypotheses.

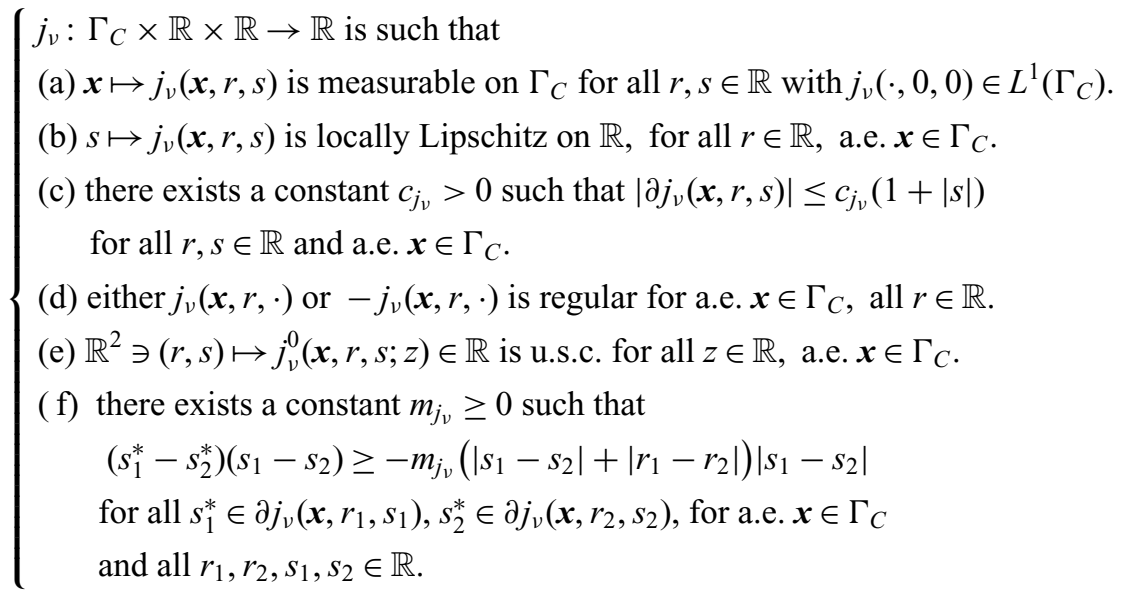


$\left(j_{\tau}: \Gamma_{C} \times \mathbb{R} \times \mathbb{R}^{d} \rightarrow \mathbb{R}\right.$ is such that

(a) $\boldsymbol{x} \mapsto j_{\tau}(\boldsymbol{x}, r, \boldsymbol{\xi})$ is measurable on $\Gamma_{C}$ for all $(r, \boldsymbol{\xi}) \in \mathbb{R} \times \mathbb{R}^{d}$

with $j_{\tau}\left(\cdot, 0, \mathbf{0}_{\mathbb{R}^{d}}\right) \in L^{1}\left(\Gamma_{C}\right)$.

(b) $\boldsymbol{\xi} \mapsto j_{\tau}(\boldsymbol{x}, r, \boldsymbol{\xi})$ is locally Lipschitz on $\mathbb{R}^{d}$, for all $r \in \mathbb{R}$, a.e. $\boldsymbol{x} \in \Gamma_{C}$.

(c) there exists a constant $c_{j_{\tau}}>0$ such that $\left\|\partial j_{\tau}(\boldsymbol{x}, r, \boldsymbol{\xi})\right\|_{\mathbb{R}^{d}} \leq c_{j_{\tau}}\left(1+\|\boldsymbol{\xi}\|_{\mathbb{R}^{d}}\right)$

for all $(r, \boldsymbol{\xi}) \in \mathbb{R} \times \mathbb{R}^{d}$ and a.e. $\boldsymbol{x} \in \Gamma_{C}$.

(d) either $j_{\tau}(\boldsymbol{x}, r, \cdot)$ or $-j_{\tau}(\boldsymbol{x}, r, \cdot)$ is regular for a.e. $\boldsymbol{x} \in \Gamma_{C}$ and $r \in \mathbb{R}$.

(e) $\mathbb{R} \times \mathbb{R}^{d} \ni(r, \boldsymbol{\xi}) \mapsto j_{\tau}^{0}(\boldsymbol{x}, r, \boldsymbol{\xi} ; \boldsymbol{\eta}) \in \mathbb{R}$ is u.s.c. for all $\boldsymbol{\eta} \in \mathbb{R}^{d}$, a.e. $\boldsymbol{x} \in \Gamma_{C}$.

(f) there exists $m_{j_{\tau}} \geq 0$ such that

$$
\left(\zeta_{1}-\zeta_{2}\right) \cdot\left(\boldsymbol{\xi}_{1}-\boldsymbol{\xi}_{2}\right) \geq-m_{j_{\tau}}\left(\left\|\boldsymbol{\xi}_{1}-\boldsymbol{\xi}_{2}\right\|_{\mathbb{R}^{d}}+\left|r_{1}-r_{2}\right|\right)\left\|\boldsymbol{\xi}_{1}-\boldsymbol{\xi}_{2}\right\|_{\mathbb{R}^{d}}
$$

for all $\zeta_{1} \in \partial j_{\tau}\left(\boldsymbol{x}, r_{1}, \boldsymbol{\xi}_{1}\right), \zeta_{2} \in \partial j_{\tau}\left(\boldsymbol{x}, r_{2}, \boldsymbol{\xi}_{2}\right)$, a.e. $\boldsymbol{x} \in \Gamma_{C}$ and all $r_{1}, r_{2} \in \mathbb{R}, \boldsymbol{\xi}_{1}, \boldsymbol{\xi}_{2} \in \mathbb{R}^{d}$.

In hypotheses (4.18) and (4.19), symbols $\partial j_{v}$ and $\partial j_{\tau}$ stand for the generalised gradient of $j_{v}$ and $j_{\tau}$, respectively, with respect to their last argument, and $j_{v}^{0}$ and $j_{\tau}^{0}$ denote the generalised directional derivatives of $j_{v}(\boldsymbol{x}, r, \cdot)$ and of $j_{\tau}(\boldsymbol{x}, r, \cdot)$.

The non-linear function $F$ is assumed to have the following properties.

$\left(F: \Gamma_{C} \times(0, T) \times \mathbb{R}^{d} \times \mathbb{R} \rightarrow \mathbb{R}\right.$ is such that

(a) $F(\cdot, \cdot, \xi, r)$ is measurable on $\Gamma_{C} \times(0, T)$ for all $(\xi, r) \in \mathbb{R}^{d} \times \mathbb{R}$.

(b) there exists a constant $L_{F}>0$ such that

$$
\left|F\left(\boldsymbol{x}, t, \boldsymbol{\xi}_{1}, r_{1}\right)-F\left(\boldsymbol{x}, t, \boldsymbol{\xi}_{2}, r_{2}\right)\right| \leq L_{F}\left(\left\|\boldsymbol{\xi}_{1}-\boldsymbol{\xi}_{2}\right\|_{\mathbb{R}^{d}}+\left|r_{1}-r_{2}\right|\right)
$$

for a.e. $(\boldsymbol{x}, t) \in \Gamma_{C} \times(0, T)$ and all $\left(\boldsymbol{\xi}_{i}, r_{i}\right) \in \mathbb{R}^{d} \times \mathbb{R}, i=1,2$.

(c) $F(\boldsymbol{x}, t, \boldsymbol{\xi}, 0)=0, F(\boldsymbol{x}, t, \boldsymbol{\xi}, r) \geq 0$ for $r \leq 0$ and $F(\boldsymbol{x}, t, \boldsymbol{\xi}, r) \leq 0$

for $r \geq 1$, for a.e. $(x, t) \in \Gamma_{C} \times(0, T)$, and for all $\xi \in \mathbb{R}^{d}$.

(d) there exists a constant $M_{F}>0$ such that $|F(x, t, \xi, r)| \leq M_{F}$ for all $(\boldsymbol{x}, t, \xi, r) \in \Gamma_{C} \times(0, T) \times \mathbb{R}^{d} \times[0,1]$.

Besides, the densities of volume forces and surface tractions satisfy

$$
\boldsymbol{f}_{0} \in H^{1}\left(0, T ; L^{2}\left(\Omega ; \mathbb{R}^{d}\right)\right), \quad \boldsymbol{f}_{N} \in H^{1}\left(0, T ; L^{2}\left(\Gamma_{N} ; \mathbb{R}^{d}\right)\right) .
$$

The initial displacement, velocity and boding field are assumed to satisfy the conditions.

$$
\boldsymbol{u}_{0} \in V, \quad \boldsymbol{v}_{0} \in K, \quad \beta_{0} \in L^{2}\left(\Gamma_{C}\right), \quad 0 \leq \beta_{0}(x) \leq 1 \text { a.e. } \boldsymbol{x} \in \Gamma_{C} .
$$

To derive the weak formulation of the dynamic contact problem, we assume that $(\boldsymbol{u}, \boldsymbol{\sigma})$ are smooth functions on $\mathcal{Q}$ such that (4.1)-(4.9) hold. Let $\boldsymbol{v} \in V$ be arbitrary and $H:=L^{2}\left(\Omega ; \mathbb{R}^{d}\right)$. We multiply the equation of motion (4.2) by $\boldsymbol{v}-\boldsymbol{u}^{\prime}(t)$ and employ the Green formula, see [35, Theorem 2.25], to obtain

$$
\left\langle\boldsymbol{u}^{\prime \prime}(t), \boldsymbol{v}-\boldsymbol{u}^{\prime}(t)\right\rangle+\left\langle\boldsymbol{\sigma}(t), \boldsymbol{\varepsilon}(\boldsymbol{v})-\boldsymbol{\varepsilon}\left(\boldsymbol{u}^{\prime}(t)\right)\right\rangle_{\mathcal{H}}=\left(\boldsymbol{f}_{0}(t), \boldsymbol{v}-\boldsymbol{u}^{\prime}(t)\right)_{H}+\int_{\partial \Omega} \boldsymbol{\sigma}(t) \boldsymbol{v} \cdot\left(\boldsymbol{v}-\boldsymbol{u}^{\prime}(t)\right) d \Gamma
$$


for a.e. $t \in(0, T)$. Define $\boldsymbol{f} \in H^{1}\left(0, T ; V^{*}\right)$ (see hypothesis (4.21)) by

$$
\langle\boldsymbol{f}(t), \boldsymbol{v}\rangle=\left(\boldsymbol{f}_{0}(t), \boldsymbol{v}\right)_{H}+\left(\boldsymbol{f}_{N}(t), \boldsymbol{v}\right)_{L^{2}\left(\Gamma_{N} ; \mathbb{R}^{d}\right)} \text { for all } \boldsymbol{v} \in V \text {, a.e. } t \in(0, T) .
$$

Then, apply boundary conditions (4.3)-(4.4) to get

$$
\left\langle\boldsymbol{u}^{\prime \prime}(t), \boldsymbol{v}-\boldsymbol{u}^{\prime}(t)\right\rangle+\left\langle\boldsymbol{\sigma}(t), \boldsymbol{\varepsilon}(\boldsymbol{v})-\boldsymbol{\varepsilon}\left(\boldsymbol{u}^{\prime}(t)\right)\right\rangle_{\mathcal{H}}=\left\langle\boldsymbol{f}(t), \boldsymbol{v}-\boldsymbol{u}^{\prime}(t)\right\rangle+\int_{\Gamma_{C}} \boldsymbol{\sigma}(t) \boldsymbol{v} \cdot\left(\boldsymbol{v}-\boldsymbol{u}^{\prime}(t)\right) d \Gamma
$$

for a.e. $t \in(0, T)$. Since

$$
\int_{\Gamma_{C}} \boldsymbol{\sigma}(t) \boldsymbol{v} \cdot \boldsymbol{v} d \Gamma=\int_{\Gamma_{C}}\left(\sigma_{v}(t) v_{v}+\boldsymbol{\sigma}_{\tau}(t) \cdot \boldsymbol{v}_{\tau}\right) d \Gamma
$$

and from boundary conditions (4.5)-(4.6),

$$
\left\{\begin{array}{l}
-\sigma_{v}(t)\left(v_{v}-u_{v}^{\prime}(t)\right) \leq j_{v}^{0}\left(\boldsymbol{x}, \beta(t), u_{v}^{\prime}(t) ; v_{v}-u_{v}^{\prime}(t)\right) \\
-\sigma_{\tau}(t) \cdot\left(\boldsymbol{v}_{\tau}-\boldsymbol{u}_{\tau}^{\prime}(t)\right) \leq j_{\tau}^{0}\left(\boldsymbol{x}, \beta(t), \boldsymbol{u}_{\tau}^{\prime}(t) ; \boldsymbol{v}_{\tau}-\boldsymbol{u}_{\tau}^{\prime}(t)\right)
\end{array}\right.
$$

on $\Sigma_{C}$, we deduce from (4.23) that

$$
\begin{gathered}
\left\langle\boldsymbol{u}^{\prime \prime}(t), \boldsymbol{v}-\boldsymbol{u}^{\prime}(t)\right\rangle+\left\langle\boldsymbol{\sigma}(t), \boldsymbol{\varepsilon}(\boldsymbol{v})-\boldsymbol{\varepsilon}\left(\boldsymbol{u}^{\prime}(t)\right)\right\rangle_{\mathcal{H}}+\int_{\Gamma_{C}} j_{\nu}^{0}\left(\boldsymbol{x}, \beta(t), u_{v}^{\prime}(t) ; v_{v}-u_{v}^{\prime}(t)\right) d \Gamma \\
+\int_{\Gamma_{C}} j_{\tau}^{0}\left(\boldsymbol{x}, \beta(t), \boldsymbol{u}_{\tau}^{\prime}(t) ; \boldsymbol{v}_{\tau}-\boldsymbol{u}_{\tau}^{\prime}(t)\right) d \Gamma \geq\left\langle\boldsymbol{f}(t), \boldsymbol{v}-\boldsymbol{u}^{\prime}(t)\right\rangle
\end{gathered}
$$

for all $v \in K$ and a.e. $t \in(0, T)$. So the weak formulation of Problem 16 is the following.

Problem 18 Find a displacement field $\boldsymbol{u}:(0, T) \rightarrow V$, and a bonding field $\beta:(0, T) \rightarrow L^{2}\left(\Gamma_{C}\right)$ such that $\boldsymbol{u} \in \mathcal{V}, \boldsymbol{u}^{\prime} \in \mathcal{W}, \beta \in W^{1,2}\left(0, T ; L^{2}\left(\Gamma_{C}\right)\right), 0 \leq \beta(t) \leq 1$ for all $t \in[0, T]$, a.e. on $\Gamma_{C}$, and

$$
\left\{\begin{array}{l}
\left\langle\boldsymbol{u}^{\prime \prime}(t), \boldsymbol{v}-\boldsymbol{u}^{\prime}(t)\right\rangle+\left\langle\mathcal{C}\left(\boldsymbol{\varepsilon}\left(\boldsymbol{u}^{\prime}(t)\right)\right)+\mathcal{E}(\boldsymbol{\varepsilon}(\boldsymbol{u}(t))), \boldsymbol{\varepsilon}(\boldsymbol{v})-\boldsymbol{\varepsilon}\left(\boldsymbol{u}^{\prime}(t)\right)\right\rangle_{\mathcal{H}} \\
\quad+\int_{\Gamma_{C}} j_{v}^{0}\left(\boldsymbol{x}, \beta(t), u_{v}^{\prime}(t) ; v_{v}-u_{v}^{\prime}(t)\right) d \Gamma+\int_{\Gamma_{C}} j_{\tau}^{0}\left(\boldsymbol{x}, \beta(t), \boldsymbol{u}_{\tau}^{\prime}(t) ; \boldsymbol{v}_{\tau}-\boldsymbol{u}_{\tau}^{\prime}(t)\right) d \Gamma \\
\quad \geq\left\langle\boldsymbol{f}(t), \boldsymbol{v}-\boldsymbol{u}^{\prime}(t)\right\rangle \text { for all } \boldsymbol{v} \in K \text { and a.e. } t \in(0, T), \\
\beta^{\prime}(t)=F(t, \boldsymbol{u}(t), \beta(t)) \quad \text { on } \Sigma_{C}, \\
\beta(0)=\beta_{0} \quad \text { on } \Gamma_{C}, \\
\boldsymbol{u}(0)=\boldsymbol{u}_{0}, \boldsymbol{u}^{\prime}(0)=\boldsymbol{v}_{0} \text { in } \Omega .
\end{array}\right.
$$

Let $X=L^{2}\left(\Gamma_{C} ; \mathbb{R}^{d}\right)$ and $Y=L^{2}\left(\Gamma_{C}\right)$. We will apply Theorems 13 and 14 to study Problem 18 . For this purpose, we introduce operators $A: V \rightarrow V^{*}$ and $B: V \rightarrow V^{*}$ defined by

$$
\begin{aligned}
\langle A(\boldsymbol{u}), \boldsymbol{v}\rangle & =\langle\mathcal{C}(\boldsymbol{\varepsilon}(\boldsymbol{u})), \boldsymbol{\varepsilon}(\boldsymbol{v})\rangle_{\mathcal{H}}, \\
\langle B(\boldsymbol{u}), \boldsymbol{v}\rangle & =\langle\mathcal{E}(\boldsymbol{\varepsilon}(\boldsymbol{u})), \boldsymbol{\varepsilon}(\boldsymbol{v})\rangle_{\mathcal{H}},
\end{aligned}
$$

for all $\boldsymbol{u}, \boldsymbol{v} \in V$, respectively. Also, we define $M: V \rightarrow X$ and $J: Y \times X \rightarrow \mathbb{R}$ by

$$
\begin{aligned}
& M=\gamma: V \rightarrow X \text { the trace operator, } \\
& J(\beta, \boldsymbol{u})=\int_{\Gamma_{C}}\left(j_{\nu}\left(\boldsymbol{x}, \beta, u_{v}\right)+j_{\tau}\left(\boldsymbol{x}, \beta, \boldsymbol{u}_{\tau}\right)\right) d \Gamma \text { for } \beta \in Y, \boldsymbol{u} \in X .
\end{aligned}
$$


Theorem 19 Assume (4.16)-(4.22). In addition, assume the compatibility condition

$$
\left(A\left(\boldsymbol{v}_{0}\right)+B \boldsymbol{u}_{0}+\gamma^{*} \partial J\left(\beta_{0}, \gamma \boldsymbol{v}_{0}\right)-\boldsymbol{f}(0)\right) \cap H \neq \emptyset,
$$

and $m_{\mathcal{C}}>\|\gamma\|^{2}\left(m_{j_{v}}+m_{j_{\tau}}\right)$. Then, Problem 18 has a unique solution $(\boldsymbol{u}, \beta)$ such that

$$
\boldsymbol{u} \in W^{1,2}(0, T ; V), \boldsymbol{u} \in L^{2}\left(0, T ; V^{*}\right), \beta \in C\left(0, T ; L^{2}\left(\Gamma_{C}\right)\right), 0 \leq \beta(t) \leq 1 \text { for all } t \in[0, T] .
$$

Proof Let $i$ be the embedding from $V$ to $H$ which is compact. Define $\varphi: V \rightarrow\{0,+\infty\}$ by

$$
\varphi(\boldsymbol{v})=I_{K}(\boldsymbol{v})= \begin{cases}0, & \text { if } \boldsymbol{v} \in K \\ +\infty, & \text { otherwise }\end{cases}
$$

where $K$ is given by (4.15). Since $K$ is a non-empty, closed and convex subset of $V$, the indicator function $\varphi=I_{K}$ satisfies $H(\varphi)$, see [24, p. 12]. Hypotheses (4.18)(d) and (4.19)(d) imply, see [35, Lemma 3.39(3) and Corollary 4.15(vi)]

$$
J^{0}(\beta, \boldsymbol{u})=\int_{\Gamma_{C}}\left(j_{\nu}^{0}\left(\boldsymbol{x}, \beta, u_{v}\right)+j_{\tau}^{0}\left(\boldsymbol{x}, \beta, \boldsymbol{u}_{\tau}\right)\right) d \Gamma,
$$

for all $\beta \in Y$ and $\boldsymbol{u} \in X$. Combining (4.26)-(4.29) with (4.31) and (4.32), we can reformulate (4.25) as

$$
\left\{\begin{aligned}
&\left\langle\boldsymbol{u}^{\prime \prime}(t), \boldsymbol{v}-\boldsymbol{u}^{\prime}(t)\right\rangle+\left\langle A\left(\boldsymbol{u}^{\prime}(t)\right)+B(\boldsymbol{u}(t)), \boldsymbol{v}-\boldsymbol{u}^{\prime}(t)\right\rangle+\varphi(\boldsymbol{v})-\varphi\left(\boldsymbol{u}^{\prime}(t)\right) \quad+J^{0}\left(\beta(t), M \boldsymbol{u}^{\prime}(t) ; M\left(\boldsymbol{v}-\boldsymbol{u}^{\prime}(t)\right)\right) \geq\left\langle\boldsymbol{f}(t), \boldsymbol{v}-\boldsymbol{u}^{\prime}(t)\right\rangle \\
& \quad \text { for all } \boldsymbol{v} \in V, \text { a.e. } t \in(0, T), \\
& \beta^{\prime}(t)=\mathcal{F}(t, \boldsymbol{u}(t), \beta(t)) \text { on } \Sigma_{C}, \\
& \beta(0)=\beta_{0} \text { on } \Gamma_{C}, \\
& \boldsymbol{u}(0)=\boldsymbol{u}_{0}, \boldsymbol{u}^{\prime}(0)=\boldsymbol{v}_{0} \text { in } \Omega,
\end{aligned}\right.
$$

where $\mathcal{F}:(0, T) \times X \times Y \rightarrow Y$ is the Nemytskii operator of $F$ defined by

$$
\mathcal{F}(t, \beta, \boldsymbol{u})(\boldsymbol{x})=F(\boldsymbol{x}, t, \beta(\boldsymbol{x}), \boldsymbol{u}(\boldsymbol{x})) \text { for a.e. } \boldsymbol{x} \in \Gamma_{C} .
$$

Furthermore, (4.33) is equivalent to, see [35, Proposition 3.23]

$$
\left\{\begin{array}{l}
\left\langle\boldsymbol{u}^{\prime \prime}(t), \boldsymbol{v}-\boldsymbol{u}^{\prime}(t)\right\rangle+\left\langle A\left(\boldsymbol{u}^{\prime}(t)\right)+B(\boldsymbol{u}(t)), \boldsymbol{v}-\boldsymbol{u}^{\prime}(t)\right\rangle+\varphi(\boldsymbol{v})-\varphi\left(\boldsymbol{u}^{\prime}(t)\right) \\
\quad+\left\langle\boldsymbol{\xi}(t), M\left(\boldsymbol{v}-\boldsymbol{u}^{\prime}(t)\right)\right\rangle_{X^{*} \times X} \geq\left\langle\boldsymbol{f}(t), \boldsymbol{v}-\boldsymbol{u}^{\prime}(t)\right\rangle \text { for all } \boldsymbol{v} \in V, \text { a.e. } t \in(0, T), \\
\boldsymbol{\xi}(t) \in \partial J\left(\beta(t), M \boldsymbol{u}^{\prime}(t)\right) \text { for a.e. } t \in(0, T), \\
\beta^{\prime}(t)=\mathcal{F}(t, \boldsymbol{u}(t), \beta(t)) \text { on } \Sigma_{C}, \\
\beta(0)=\beta_{0} \text { on } \Gamma_{C}, \\
\boldsymbol{u}(0)=\boldsymbol{u}_{0}, \boldsymbol{u}^{\prime}(0)=\boldsymbol{v}_{0} \text { in } \Omega .
\end{array}\right.
$$

We now verify the conditions stated in Theorem 13. The condition (4.16)(d) guarantees that $A$ is strongly monotone with constants $m_{A}=m_{\mathcal{C}}$ and $c_{A}=0$. Hypotheses (4.16)(b) and (c) imply 
that $A$ is continuous and bounded. Therefore, it is pseudomonotone, see [35, Theorem 3.69], that is, $H(A)(\mathrm{i})$ is valid. The growth condition $(4.16)(\mathrm{c})$ entails

$$
\begin{aligned}
\|A \boldsymbol{v}\|_{V^{*}} & =\sup _{\|\boldsymbol{w}\|=1, \boldsymbol{w} \in V}|\langle A \boldsymbol{v}, \boldsymbol{w}\rangle| \leq \int_{\Omega}\|\mathcal{C}(\boldsymbol{x}, \boldsymbol{\varepsilon}(\boldsymbol{v}))\|_{\mathbb{S}^{d}}\|\boldsymbol{\varepsilon}(\boldsymbol{w})\|_{\mathbb{S}^{d}} d \boldsymbol{x} \\
& \leq\left(\int_{\Omega}\left(b_{\mathcal{C}}(\boldsymbol{x})+c_{\mathcal{C}}\|\boldsymbol{\varepsilon}(\boldsymbol{v})\|_{\mathbb{S}^{d}}\right)^{2} d \boldsymbol{x}\right)^{\frac{1}{2}} \leq \sqrt{2}\left(\left\|b_{\mathcal{C}}\right\|_{L^{2}(\Omega)}+c_{\mathcal{C}}\|\boldsymbol{v}\|\right) .
\end{aligned}
$$

This implies that $A$ satisfies $H(A)$ (ii) with $a_{A}=\sqrt{2}\left\|b_{\mathcal{C}}\right\|_{L^{2}(\Omega)}$ and $b_{A}=\sqrt{2} c_{\mathcal{C}}$. As concerns operator $B$, it is easy to see that under hypothesis (4.17), $B$ defined by (4.27) satisfies $H(B)$ with $\varrho_{B}=m_{\mathcal{E}}$.

By (4.18)(a)-(c) and (4.19)(a)-(c), we know that the function $J$ defined by (4.29) satisfies properties $H(J)(\mathrm{i})$ and (ii), see [35, Corollary 4.15]. The assumptions (4.18)(e) and (4.19)(e) show that either $J(\beta, \cdot)$ or $-J(\beta, \cdot)$ is regular on $X$ for all $\beta \in Y$, see [35, Corollary 4.15(vii)]. Thus, by [35, Lemma 3.39(3) and Corollary 4.15(vi)]), we have

$$
\partial J(\beta, \boldsymbol{u})=\int_{\Gamma_{C}}\left(\partial j_{v}\left(\boldsymbol{x}, \beta, u_{v}\right)+\partial j_{\tau}\left(\boldsymbol{x}, \beta, \boldsymbol{u}_{\tau}\right)\right) d \Gamma .
$$

The upper semicontinuity of $j_{v}$ and $j_{\tau}$ combined with the Fatou's lemma, see [35, Theorem 1.64] implies that $(\beta, \boldsymbol{u}) \mapsto J^{0}(\beta, \boldsymbol{u} ; \boldsymbol{v})$ is upper semicontinuous on $Y \times X$ for all $\boldsymbol{v} \in X$. Hence, $H(J)(\mathrm{iv})$ holds. The relaxed monotonicity of $\partial J$ (cf. $H(J)(\mathrm{iii}))$ is obtained using (4.18)(f), (4.19)(f), (4.36) and the Hölder's inequality.

By the Sobolev trace theorem, see, for example, $[10,35,48]$, we know that the operator $M: V \rightarrow X$ is linear and continuous. To check condition $H(M)$, let $\left\{\boldsymbol{w}_{n}\right\}$ be a sequence bounded in $M^{2,2}\left(0, T ; V, V^{*}\right)$ and $\delta \in(0,1 / 2)$. The embedding $M^{2,2}\left(0, T ; V, V^{*}\right) \subset L^{2}\left(0, T ; H^{1 / 2+\delta}\left(\Omega ; \mathbb{R}^{d}\right)\right)$ is compact by Proposition 2. Hence, there exists a subsequence $\left\{\boldsymbol{w}_{n_{k}}\right\}$ of $\left\{\boldsymbol{w}_{n}\right\}$ such that $\boldsymbol{w}_{n_{k}} \rightarrow$ $\boldsymbol{w}$ in $L^{2}\left(0, T ; H^{1 / 2+\delta}\left(\Omega ; \mathbb{R}^{d}\right)\right)$ for some element $\boldsymbol{w} \in L^{2}\left(0, T ; H^{1 / 2+\delta}\left(\Omega ; \mathbb{R}^{d}\right)\right)$. Since the trace operator from $L^{2}\left(0, T ; H^{1 / 2+\delta}\left(\Omega ; \mathbb{R}^{d}\right)\right)$ to $L^{2}\left(0, T ; H^{\delta}\left(\Gamma_{C} ; \mathbb{R}^{d}\right)\right) \subset L^{2}(0, T ; X)=\mathcal{X}$ is linear and continuous, we deduce that $\mathcal{M} \boldsymbol{w}_{n_{k}} \rightarrow \mathcal{M} \boldsymbol{w}$ in $\mathcal{X}$. Thus, condition $H(M)$ is satisfied.

Next, the compatibility conditions (4.30) and (4.22) imply $\mathbf{0}_{V^{*}} \in \partial_{c} I_{K}\left(\boldsymbol{v}_{0}\right)$. So we are able to find $\boldsymbol{z}_{0} \in H$ such that

$$
\boldsymbol{z}_{0} \in A\left(\boldsymbol{v}_{0}\right)+B \boldsymbol{u}_{0}+\gamma^{*} \partial J\left(\beta_{0}, \gamma \boldsymbol{v}_{0}\right)-\boldsymbol{f}(0)
$$

This result and the smallness condition $m_{\mathcal{C}}>\|\gamma\|^{2}\left(m_{j_{v}}+m_{j_{\tau}}\right)$ imply $H(0)$. It is easy to demonstrate, by hypothesis (4.20), that the function $\mathcal{F}$ defined in (4.34) satisfies $H(F)$. Finally, from hypothesis (4.20)(c), by an analogous proof as in [33, Lemma 5], we deduce that $\beta(t) \in[0,1]$ for all $t \in[0, T]$, a.e. on $\Gamma_{C}$.

The conclusion of Theorem 19 now follows from an application of Theorems 13 and 14.

Finally, we make a comment on the validity of the compatibility condition (4.30). Assume

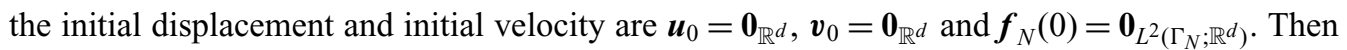
hypotheses (4.16)(e) and (4.17) imply $A \boldsymbol{v}_{0}=B \boldsymbol{u}_{0}=\mathbf{0}_{V^{*}}$. Since $\mathbf{0}_{X^{*}} \in \partial J\left(\gamma \mathbf{0}_{V}\right)$ and $\boldsymbol{v}_{0} \in K$ (due to $\boldsymbol{v}_{0} \cdot \boldsymbol{v}=0 \leq g$ ), the compatibility condition (4.30) is valid in this case. 


\section{References}

[1] Atkinson, K. \& HAN, W. (2009) Theoretical Numerical Analysis: A Functional Analysis Framework, 3rd ed., Springer-Verlag, New York.

[2] Aubin, J. P. \& Cellina, A. (1984) Differential Inclusions. Set-Valued Maps and Viability Theory, Springer-Verlag, Berlin, Heidelberg, New York, Tokyo.

[3] BARtosz, K. (2018) Convergence of Rothe scheme for a class of dynamic variational inequalities involving Clarke subdifferential. Appl. Anal. 97, 2189-2209.

[4] Bartosz, K., Cheng, X., Kalita, P., YU, Y. \& Zheng, C. (2015) Rothe method for parabolic variational-hemivariational inequalities. J. Math. Anal. Appl. 423, 841-862.

[5] BARTOSZ, K. \& SofonEA, M. (2016) The Rothe method for variational-hemivariational inequalities with applications to contact mechanics. SIAM J. Math. Anal. 48, 861-883.

[6] Carl, S., Le, V. K. \& Motreanu, D. (2007) Nonsmooth Variational Problems and Their Inequalities: Comparison Principles and Applications, Springer, New York.

[7] Carstensen, C. \& Gwinner, J. (1999) A theory of discretization for nonlinear evolution inequalities applied to parabolic Signorini problems. Ann. Mat. Pura Appl. 177, 363-394.

[8] Clarke, F. H. (1983) Optimization and Nonsmooth Analysis, Wiley, Interscience, New York.

[9] Denkowski, Z., Migórski, S. \& Papageorgiou, N.S. (2003) An Introduction to Nonlinear Analysis: Theory, Kluwer Academic/Plenum Publishers, Boston, Dordrecht, London, New York.

[10] Denkowski, Z., Migórski, S. \& Papageorgiou, N. S. (2003) An Introduction to Nonlinear Analysis: Applications, Kluwer Academic/Plenum Publishers, Boston, Dordrecht, London, New York.

[11] Duvaut, G. \& Lions, J. L. (1976) Inequalities in Mechanics and Physics, Springer, Berlin.

[12] ECK, C., JARUŠEK, J. \& KRBEČ, M. (2005) Unilateral Contact Problems: Variational Methods and Existence Theorems, Pure and Applied Mathematics, Vol. 270, Chapman/CRC Press, New York.

[13] ECK, C., JARUŠEK, J. \& SOFONEA, M. (2010) A dynamic elastic-visco-plastic unilateral contact probelm with normal damped response and Coulomb friction. European J. Appl. Math. 21, 229-251.

[14] Frémond, M. (1982) Equilibre des structures qui adhérent á leur support. C.R. Acad. Sci. Paris 295, 913-916.

[15] FrÉmond, M. (1987) Adhérence des solides. J. Méchanique Théorique et Appliquée 6, 383-407.

[16] FrÉmond, M. (2012) Phase Change in Mechanics, Springer-Verlag, Berlin.

[17] HAN, J. F., LI, Y. X. \& MigÓRSKI, S. (2015) Analysis of an adhesive contact problem for viscoelastic materials with long memory. J. Math. Anal. Appl. 427, 646-668.

[18] HAN, J. F., MigóRSKI, S. \& ZENG, H. D. (2016) Analysis of a dynamic viscoelastic unilateral contact problem with normal damped response. Nonlinear Anal. 28, 229-250.

[19] Han, W., Migórski, S. \& Sofonea, M. (2014) A class of variational-hemivariational inequalities with applications to frictional contact problems. SIAM J. Math. Anal. 46, 3891-3912.

[20] Han, W., Migórski, S. \& Sofonea, M. (editors) (2015) Advances in Variational and Hemivariational Inequalities with Applications. Theory, Numerical Analysis, and Applications, Advances in Mechanics and Mathematics, Vol. 33, Springer, Cham, Heidelberg, New York.

[21] Han, W., Shillor, M. \& Sofonea, M. (2001) Variational and numerical analysis of a quasistatic viscoelastic problem with normal compliance, friction and damage. J. Comp. Appl. Math. 137, 377-398.

[22] Han, W. \& Sofonea, M. (2002) Quasistatic Contact Problems in Viscoelasticity and Viscoplasticity, Studies in Advanced Mathematics, Vol. 30, Americal Mathematical Society, Providence, RI, International Press, Somerville, MA.

[23] Han, W., Sofonea, M. \& Danan, D. (2018) Numerical analysis of stationary variationalhemivariational inequalities. Numer. Math. 139, 563-592.

[24] Haslinger, J., Miettinen, M. \& Panagiotopoulos, P. D. (1999) Finite Element Method for Hemivariational Inequalities. Theory, Methods and Applications, Kluwer Academic Publishers, Boston, Dordrecht, London. 
[25] Kalita, P. (2012) Regularity and Rothe method error estimates for parabolic hemivariational inequality. J. Math. Anal. Appl. 389, 618-631.

[26] Kalita, P. (2013) Convergence of Rothe scheme for hemivariational inequalities of parabolic type. Int. J. Numer. Anal. Model. 10, 445-465.

[27] KikUChI, N. \& Oden, J. T. (1988) Contact Problems in Elasticity: A Study of Variational Inequalities and Finite Element Methods, SIAM, Philadelphia.

[28] Kinderlehrer, D. \& Stampacchia, G. (2000) An Introduction to Variational Inequalities and Their Applications, Classics in Applied Mathematics, Vol. 31, SIAM, Philadelphia.

[29] LE, V. K. (2011) Range and existence theorem for pseudomonotone perturbations of maximal monotone operators. Proc. Amer. Math. Soc. 139, 1645-1658.

[30] LiU, Z. H., MigóRski, S. \& Zeng, S. D. (2017) Partial differential variational inequalities involving nonlocal boundary conditions in Banach spaces. J. Differ. Equations 263, 3989-4006.

[31] LiU, Z. H., Zeng, S. D. \& Motreanu, D. (2016) Evolutionary problems driven by variational inequalities. J. Differ. Equations 260, 6787-6799.

[32] LiU, Z. H. \& Motreanu, D. (2010) A class of variational-hemivariational inequalities of elliptic type. Nonlinearity 23, 1741-1752.

[33] MigóRSKI, S. \& OCHAL, A. (2008) Dynamic bilateral contact problem for viscoelastic piezoelectric materials with adhesion. Nonlinear Anal. 69, 495-509.

[34] MigÓRSKI, S. \& OCHAL, A. (2009) Quasistatic hemivariational inequality via vanishing acceleration approach. SIAM J. Math. Anal. 41, 1415-1435.

[35] Migórski, S., OChal, A. \& Sofonea, M. (2013) Nonlinear Inclusions and Hemivariational Inequalities. Models and Analysis of Contact Problems, Advances in Mechanics and Mathematics, Vol. 26, Springer, New York.

[36] MigóRSKI, S. \& ZENG, S. D. (2018) Hyperbolic hemivariational inequalities controlled by evolution equations with application to adhesive contact model. Nonlinear Anal. 43, 121-143.

[37] MigÓRSKI, S. \& ZENG, S. D. (2018) A class of differential hemivariational inequalities in Banach spaces. J. Global Optim. 72, 761-779.

[38] MigÓRSKI, S. \& ZENG, S. D. (2019) Mixed variational inequalities driven by fractional evolution equations. ACTA Math. Sci. 39, 461-468.

[39] Naniewicz, Z. \& Panagiotopoulos, P. D. (1995) Mathematical Theory of Hemivariational Inequalities and Applications, Marcel Dekker, Inc., New York, Basel, Hong Kong.

[40] Panagiotopoulos, P. D. (1983) Nonconvex energy functions, hemivariational inequalities and substationary principles. Acta Mechanica 42, 160-183.

[41] Panagiotopoulos, P. D. (1993) Hemivariational Inequalities, Applications in Mechanics and Engineering, Springer-Verlag, Berlin.

[42] Panagiotopoulos, P. D. \& Pop, G. (1999) On a type of hyperbolic variational-hemivariational inequalities. J. Appl. Anal. 5, 95-112.

[43] Shillor, M., Sofonea, M. \& Telega, J. J. (2004) Models and Analysis of Quasistatic Contact, Lecture Notes in Physics, Vol. 655, Springer, Berlin, Heidelberg.

[44] Sofonea, M., Han, W. \& Shillor, M. (2006) Analysis and Approximation of Contact Problems with Adhesion or Damage, Chapman \& Hall/CRC, Boca Raton.

[45] SofoneA, M. \& MateI, A. (2012) Mathematical Models in Contact Mechanics, London Mathematical Society Lecture Note Series, Vol. 398, Cambridge University Press.

[46] SofoneA, M. \& MigóRSKI, S. (2018) Variational-Hemivariational Inequalities with Applications, Chapman \& Hall/CRC Press, Boca Raton, London.

[47] Sofonea, M., RENON, N. \& Shillor, M. (2004) Stress formulation for frictionless contact of an elastic-perfectly-plastic body. Appl. Anal. 83 (11), 1157-1170.

[48] Zeidler, E. (1990) Nonlinear Functional Analysis and Applications II A/B, Springer, New York.

[49] ZENG, S. D., LIU, Z. H. \& MigóRSKI, S. (2018) A class of fractional differential hemivariational inequalities with application to contact problem. Z. Angew. Math. Phys. 69(36), 23. 